\title{
Mapping the architecture of regulatory variation provides insights into the evolution of complex traits
}

Offir Lupo ${ }^{1}$, Gat Krieger ${ }^{1,2}$, Felix Jonas ${ }^{1}$ and Naama Barkai ${ }^{1^{*}}$

${ }^{1}$ Department of Molecular Genetics, Weizmann Institute of Science, Rehovot 76100, Israel

${ }^{2}$ Department of Plant and Environmental Sciences, Weizmann Institute of Science, Rehovot

76100, Israel

*Corresponding author: naama.barkai@weizmann.ac.il 


\begin{abstract}
Background: Organisms evolve complex traits by recruiting existing programs to new contexts, referred as co-option. Within a species, single upstream regulators can trigger full differentiation programs. Distinguishing whether co-option of differentiation programs results from variation in single regulator, or in multiple genes, is key for understanding how complex traits evolve. As an experimentally accessible model for studying this question we turned to budding yeast, where a differentiation program (filamentous) is activated in S. cerevisiae only upon starvation, but used by the related species $S$. paradoxus also in rich conditions.
\end{abstract}

Results: To define expression variations associated with species-specific activation of the filamentous program, we profiled the transcriptome of S. cerevisiae, S. paradoxus and their hybrid along two cell cycles at 5-minutes resolution. As expected in cases of co-option, expression of oscillating genes varies between the species in synchrony with their growth phenotypes and was dominated by upstream trans-variations. Focusing on regulators of filamentous growth, we identified gene-linked variations (cis) in multiple genes across regulatory layers, which propagated to affect expression of target genes, as well as binding specificities of downstream transcription factor. Unexpectedly, variations in regulators essential for $S$. cerevisiae filamentation were individually too weak to explain activation of this program in S. paradoxus.

Conclusions: Our study reveals the complex architecture of regulatory variation associated with species-specific use of a differentiation program. Based on these results, we suggest a new model in which evolutionary co-option of complex traits is stabilized in a distributed manner through multiple weak-effect variations accumulating throughout the regulatory network.

\title{
Introduction
}

New phenotypes arise in evolution from mutations that change gene function or regulation. At the molecular level, the majority of mutations are neutral or of small phenotypic effects[1]. Still, evolutionary related species that express the same set of proteins differ in complex phenotypes, 
including size, growth pattern, and body morphology[2-4]. A compelling model is that complex traits do not evolve de-novo but emerge through recruitment (co-option) of existing gene expression program to new contexts. Evidence supporting co-option were presented in the context of morphological evolution, where major interspecies differences in the positioning of body appendages or wing patterns were linked to variations in cis-regulatory elements controlling the expression of major regulators, including homeobox transcription factors or developmental signaling proteins[5-10].

Modulating the expression of single upstream regulators provides a genetic shortcut for the evolution of complex traits[11]. Complicating this view, however, is the fact that gene expression networks, such as these activating complex traits, are polygenic and include multiple activators and inhibitors that could act as drivers[12]. Further, due to the inter-connected nature of genetic circuits, variations in seemingly distant genes could propagate to influence the same phenotype[13]. This was exemplified recently in Drosophila, where expression variation in a Hox gene that correlated with morphological differences was found to be of little phenotypic consequence due to its masking by variations in other genes within the same pathway[14]. Understanding the genetic basis of complex traits therefore requires expanding our view from single regulators to full genetic circuits. In particular, it raises the question of whether co-option of regulatory programs result from variation in a single upstream regulator, or from multiple variations distributed among different genes. This question is difficult to address using the existing multicellular models of co-option, whose complexity precludes systematic analysis of all regulators.

As a model to address this question, we turned to budding yeast, which provides an experimentally accessible model for revealing the architecture of genetic variations associated with phenotypic differences. In multicellular models, co-option refers to the recruitment of existing differentiation programs to a new spatial-temporal context $[15,16]$. A unicellular analog of this process is the recruitment of existing differentiation programs to a new set of environmental conditions. Budding yeast undergo a dimorphic transition between yeast-form and filamentous growth modes, presenting two alternative growth programs which differ in multiple properties, including the selected budding site, cell morphology and adhesion, and the relative lengths of the different cell cycle phases[17]. In the model yeast Saccharomyces cerevisiae, transition to filamentous growth occurs mostly in nutrient-poor environments, 
allowing non-motile cells to forage for nutrients[17-19]. By contrast, S. paradoxus (CBS432), a close relative of $S$. cerevisiae, becomes filamentous even in rich media[20,21]. Therefore, the two species activate distinct differentiation programs when presented with the same condition, providing a model for evolutionary recruitment.

In addition to allowing systematic and rapid analysis, the budding yeast model offers additional key advantages for studying the basis of evolutionary recruitment. First, studies in S. cerevisiae characterized the regulatory circuits triggering the yeast to filamentous growth transition, identifying hundreds of genes that affect it and can therefore act as potential drivers of this adapted-response[22-28]. Moreover, budding yeast can mate to form inter-specific hybrids. Profiling allele-specific expression in hybrids allows to systematically distinguish variations that are linked to the gene itself ( $c i s$ ) from the that propagate from upstream variations (trans), a key for defining the genetic basis of expression divergence[29-38]. This is because within the hybrid, the two genomes are subject to the same trans environment, so that differences in the expression of the two alleles must result from cis effects. Making this distinction is particularly relevant in the case of co-option, where the cis-variations driving recruitment are expected to lead to multitude of propagating trans effects.

Our goal in this study was to reveal the architecture of expression variation underlying the differences in growth phenotypes of S. cerevisiae and S. paradoxus when growing in the same rich conditions. To this end, we examined how genes and cellular processes involved in the yeast-tofilamentous transition are expressed in the two species. By profiling the cell-cycle transcription programs of the two species and the allele-specific expression within their hybrid, we found that central pathways regulating the yeast-to-filamentous transition accumulated cis effects that bias the expression of regulators in the direction favoring the selected program. These were found in the main signal transduction components, transcription factors (TFs) and their downstream target genes. Similarly, we found variations in cell cycle TFs that propagated through a combination of cis and trans effects to influence both the expression of target genes and promoter binding specificities of another TF. Single gene candidates, tested by either deletion or promoter-swap, were insufficient to fully account for the phenotypic inter-species differences on their own. Our results suggest a model whereby co-option of differentiation programs is stabilized through the distributed action of multiple small-effects acting at different steps of the multi-layered response. 


\section{Results}

\section{Evolutionary related budding yeast species employ distinct growth program when grown in the same condition}

In rich conditions, S. cerevisiae cells grow in yeast-form. S. paradoxus, a species closely related to S. cerevisiae, appears filamentous when grown in those same conditions. Using live microscopy, we verified that S. paradoxus cells display the hallmarks of filamentous growth: increase in cell length, reorganization of budding polarity, lack of mother-daughter separation and enhanced cell to cell adhesion (Figs $1 \mathrm{~A}$ and $1 \mathrm{~B}$ ).

The changes associated with transition between yeast-form and filamentous growth, such as cell shape, budding pattern and phases duration are inherently coupled to cell cycle regulation $[17,18]$. We therefore asked whether the gene expression program of the two species is coordinated with their distinct cell cycle program. We grew diploids of both species in rich media, arrested them in early S-phase using hydroxyurea (HU), and profiled their gene expression at five minutes intervals following release from the arrest for three hours, during which they underwent two full cell-cycles (Fig 1C). Synchronized progression of the cells was validated by measuring the DNA content (Figs 1D and S1A)

Previous studies in S. cerevisiae defined the set of genes showing periodic expression along the cell cycle[39-41]. We assembled this list of cell-cycle regulated genes, and examined their dynamics along our time courses. The majority of these genes were expressed periodically in both species (72\% and 65\% in S. cerevisiae and S. paradoxus, respectively, Figs S1B-D). Further, coexpression patterns between orthologs showed high correlations (Fig 1D), confirming an overall conserved cell cycle transcription program. Classifying the genes to groups based by their cellcycle expression time, revealed periodicity that was in synchrony with cell-cycle phases, as defined by DNA content (Fig 1E). In particular, the G2-M delay and short G1 characteristics of the S. paradoxus cycle were evident in the expression of genes activated at these phases. For example, S-phase genes followed G1 genes at 10 minutes delay in S. paradoxus, compared to 20 minutes delay in S. cerevisiae (Fig 1E). Accordingly, similarity between expression profiles of the two species followed the cell cycle phase, rather than sampling time (S1E Fig). 
The global synchronization of gene expression with the cell division cycle is indicative of a global dynamic guided by variations in upstream trans regulators. We tested this by profiling the interspecific hybrid, where both genomes are subject to the same trans environment. The hybrid grew as yeast, indicating the dominance of this program (Fig 1B). Consistent with previous report[20], it progressed faster through the cell cycle, showing $5 \%$ and $15 \%$ faster growth than $S$. cerevisiae and S. paradoxus, respectively. Expression of periodic genes within the hybrid was synchronized with cell-cycle progression, with the two genomes following the same dynamics (Fig $1 \mathrm{E})$, therefore regulated in trans. Furthermore, comparing the expression levels of periodic genes revealed that trans effects dominate also variations in expression levels in addition to expression dynamics (S1F Fig). Therefore, the majority of variations in the expression of cell cycle genes propagates from variations in upstream acting trans factor(s).

\section{Cis-effects bias expression of yeast-to-filamentous regulators}

In S. cerevisiae, ectopic expression or deletion of specific regulators can induce filamentous growth independently of growth conditions[17,18]. This includes changes in the main signaling pathways, cell cycle regulation or in other genes that have an effect on the filamentous growth phenotype (Fig 2A). We reasoned that variations in regulators expression could also account for the inter-specific differences in growth modes. To systematically search for such variations, we assembled lists of genes which deletions or over-expressions affect the yeast-filamentous transition in S. cerevisiae[42] (see methods). Comparing the average expression of these genes along our time course, we found a bias favoring the respective growth mode (S2A Fig). Specifically, genes that have a negative effect on filamentous growth were more abundant in S. cerevisiae, while those having a positive effect were more abundant in S. paradoxus. Surprisingly, this bias was maintained in the hybrid, indicating its cis origin.

We next examined the two main signaling pathways that control filamentous growth, MAPK and cAMP-PKA. The MAPK cascade activates Tec1, the transcription factor (TF) acting as a master regulator of the filamentous program. The second pathway, the cAMP-PKA cascade, activates the TF Flo8 and inhibits the general co-repressors Tup1-Cyc8. In both pathways, repressors were expressed to higher levels in S. cerevisiae (Figs 5B and 5C; 5 out of the 5 differentially expressed repressors), while activators in MAPK pathways were more highly expressed in S. paradoxus (Fig 
5C 5/6). Activators in the cAMP pathway did not show any bias (Fig 5B 4/10). While most expression variations were small, expression of TEC1 stood out as being 4-folds higher in $S$. paradoxus, while its inhibitors, DIG1 and DIG2, were expressed at higher levels in S. cerevisiae (Fig 5C; 2.6 and 1.5 folds, respectively). Further, comparing alleles expression within the hybrid revealed that in the majority of genes variations result from a combination of cis and trans effects, mostly acting in the same direction (18/20), promoting the selective phenotype.

Overexpression of TEC1 in S. cerevisiae can induce filamentous growth, while its deletion prevents this transition even in nitrogen starved cells $[23,43]$. In order to see whether the changes between the species are seen also between S. cerevisiae in yeast or filamentous growth, we examined an expression dataset comparing S. cerevisiae tec1 $\triangle$ to TEC1 overexpression[24]. Indeed, the majority of expression differences between the species was similar to changes between $S$. cerevisiae in yeast-form growth and filamentous growth (S2B Fig). We therefore hypothesized that the variations in the expression of Tec1-inducing MAPK pathway genes, and in TEC1 itself, may account for S. paradoxus filamentous phenotype. To examine that, we first asked whether Tec1 target genes (Defined from[24], Fig S2C) are expressed at higher levels in S. paradoxus. This was indeed the case, as these genes showed, on average, 1.25-fold higher expression in $S$. paradoxus (Fig 2D). We expected this expression difference to be lost in the hybrid, where Tec1 does not distinguish between alleles. This, however, was not the case, as the hybrid showed 1.2fold higher expression from the S. paradoxus genome (Fig 2D), indicating on accumulated cis variations in Tec1-target genes. Further, deleting TEC1 did not reduce the expression of these genes in S. paradoxus (S2D Fig), indicating that cis expression variations in Tec1-targets were mostly independent of Tec1 itself. Consistent with that, TEC1-deleted cells showed no detectable effect on the filamentous phenotype (Fig 2E). Therefore, while variations in TEC1 correlate with S. paradoxus filamentous growth, Tec1 is not required for generating this phenotype.

The finding that Tec1 is dispensable for filamentous growth in S. paradoxus was surprising to us, as this TF is the central output of the MAPK cascade triggering filamentous growth in nutrientstarved S. cerevisiae cells. We therefore tested two additional TFs, Phd1 and Rim101, that control filamentous growth in S. cerevisiae, but are activated through pathways other than the MAPK cascade[44,45]. Neither PHD1, nor RIM101 were essential for filamentous growth in S. paradoxus (S2E Fig), though a mild reduction in flocculation was apparent. Therefore, key TFs which are 
essential for triggering filamentous growth in S. cerevisiae under poor conditions, are dispensable for this phenotype in S. paradoxus growing in rich conditions.

\section{Cis-effects bias expression of cell-cycle regulators}

The finding that key upstream activators of the filamentous program are not required for maintaining this program in rich-media growing S. paradoxus, suggests that additional variations exist, acting downstream or in parallel to these signaling pathways. In S. cerevisiae, mutating cell cycle regulators can induce filamentous growth in a manner that is independent of Tec1[24,27,46]. Variations in cell-cycle regulators could therefore contribute to the yeastfilamentous divergence of the two species (Fig 3A). To examine this, we set out to compare the expression of cell-cycle regulators between the two species.

Cell cycle progression is driven by the periodic activation of the cyclin proteins[47]. We examined the expression patterns of the three G1 cyclins (CLN1-3) which act at the G1/S transition, and the six B-type cyclins (CLB1-6) acting in the later cell cycle phases (Fig 3B). Comparing expression levels revealed that six of the nine cyclins are differentially expressed between species. These interspecies differences in expression correlate with known cell-cycle phenotypes: the longer duration of the G2/M phase of S. paradoxus for example, corresponds to the reported consequences in $S$. cerevisiae of ectopically expressing CLB3 and CLB6, or deleting CLB1[48]. Similarly, CLN3, a principle driver of the G1/S transition, showed two-fold higher expression in S. paradoxus, consistent with its shorter G1. Examining the hybrid, we found that most variations are suppressed in this uniform trans environment (Fig 3B). Therefore, while cyclins expression correlates with the respective cell-cycle phenotype, these differences are not due to cis effects, but result from trans effects propagated from variations in upstream regulators.

We next considered the TFs regulating the periodic expression of cell-cycle genes (Fig 3C). Differential expression was observed for MBP1 (FC=2), which acts at the $\mathrm{G} 1 / \mathrm{S}$ transition, and for FKH1 and FKH2 (FC=1.4, 2, respectively), which act at S/G2. All three TFs showed higher expression in S. cerevisiae. For MBP1 and FKH2, this difference in allele expression remained in the hybrid, pointing to cis variation. Testing reported targets of Fkh1 and Fkh2[46], we found that these are also more highly expressed in S. cerevisiae (average FC $=1.25$ ), but were similarly expressed in the 
hybrid, as expected of trans-effects (Fig 3D, Fig S3A). Among these targets are the cell cycle paralogs TFs Ace2 and Swi5, which regulate the expression of late M-early G1 genes. Similar to other Fkh2 targets, expression of SWI5 and ACE2 was higher S. cerevisiae (FC $=1.75,1.2$, respectively), as was the expression of Ace2's reported targets[49] (average FC= 2.6) but not Swi5's (Fig 3D, Fig S2B). These effects were mostly lost in the hybrid, indicating their trans origin (Fig 3D). Together, these results suggest that cis variations in FKH2 might propagate to affect not only its own targets, but also targets of the downstream TF, Ace2.

The transcription cascade starting in Fkh1-Fkh2 and propagating through Ace2-Swi5 is central for the transition between yeast and filamentous growth[18]. In particular, Ace2 and Swi5 induce cytokinesis by expressing cell separation genes required for the full separation of mother and daughter cells during yeast-form growth[50,51]. Further, Ace 2 inhibits the expression of CLN3 and by this extends the $\mathrm{G} 1$ duration of daughter cells, leading to the a daughter-specific delay characterizing yeast-form growth[49,52](Fig 3E). Indeed, deletion of ACE2 in S. cerevisiae reduced the portion of cells found in G1 and induces clump formation, as indicated by DNA staining (Fig 3F). Surprisingly, SWI5 deletion increased G1 duration in S. paradoxus and hybrid, but had no effect in S. cerevisiae (Fig 3F). Deletion of neither gene, however, lead to a switch in the growthmode.

Fkh1 and Fkh2 contribute to yeast growth not only by inducing ACE2 and SWI5 expression, but also by activating expression of the CLB2-gene cluster, known to influence the transition between yeast and filamentous growth[53]. We therefore asked whether the lower expression of FKH1 and FKH2 in S. paradoxus accounts for its filamentous phenotype. S. cerevisiae cells deleted of both Fkh1 and Fkh2 became filamentous even in rich conditions, consistent with previous reports[46,53] (Fig 3G). However, expressing FKH2 using the S. paradoxus promoter was sufficient for retrieving yeast growth to the FKH1-FKH2 deleted cells (Fig 3G), although FKH2 expression levels were 2-folds lower then when expressed using the S. cerevisiae promoter (S3C Fig). Therefore, the low FKH1 and FKH2 expression cannot, by itself, account for S. paradoxus filamentous phenotype. 


\section{Cis-effects bias expression of transcription-factor cascade regulating cell-separation}

Low-level expression of FKH2 is therefore sufficient for retrieving yeast growth for S. cerevisiae, but not for S. paradoxus. It was previously reported that Fkh1 and Fkh2 bind to Ace 2 target promoters and function as Swi5-specific anti-activators[54]. We therefore hypothesized that the lower expression levels of FKH1 and FKH2 may alter the activity of Swi5 and Ace2. As a measure of this activity, we measured the TFs binding profiles via ChEC-seq[55] in both species and hybrid, and examined whether binding specificities are conserved. We began this analysis with Ace2, considering its direct role in regulating cell separation after cytokinesis.

Binding profiles highly correlated between repeats ( $R=0.94-0.97$ on average), and were localized upstream to the transcription start site (TSS; S4A and S4B Figs). While the preferred Ace2 binding motif in both species was identical to the known Ace2 motif (S4C Fig; CCAGC[56]), the overall promoter selection has changed (Fig 4A top; promoter selection correlation=0.61). More specifically, promoters of cell-separation genes (CTS1, DSE1, DSE2, DSE4 and SCW11, reviewed in[50]) were bound by Ace2 more strongly in S. cerevisiae than in S. paradoxus (Fig 4A bottom; 1.8-fold higher signal on average). Notably, EGT2, the only Swi5-regualted cell separation gene[50], did not conform to this general behavior and showed higher binding signal in $S$. paradoxus. Therefore, the reduced expression of cell separation genes in S. paradoxus is not only due to the lower ACE2 levels, but also due to changes in its binding specificity between the two species.

We hypothesize that the higher expression level of the ACE2 in S. cerevisiae may affect its binding specificity. To test this, we first reduced ACE2 expression in a diploid S. cerevisiae by deleting one of its alleles, reaching levels even lower than observed in S. paradoxus. This, however, had little effect on its binding specificity ( $55 A$ Fig; $R=0.94$ ). Similarly, within the hybrid, the two Ace2 orthologs were localized to the precise same locations (S5B Fig; $R=0.99$ ). Therefore, differences in Ace 2 binding specificity in the two species are not due to differences in Ace2 expression level or protein sequence.

Binding specificity of Ace2 diverged significantly between species ( $R=0.61$, Fig $4 A$ ). We therefore asked whether these variations result from cis mutations, or from variations in a trans acting factor. To examine this, we compared Ace2 binding to the two genomes within the hybrid (Fig 4B 
top). The majority of differences in Ace2 promoter binding specificities were suppressed within the hybrid, indicating on trans acting variation (Fig $4 \mathrm{~B} ; \mathrm{R}=0.9$ ). In particular, cell separation alleles of both S. cerevisiae and S. paradoxus were bound by Ace2 to a similar extent (Fig 4B bottom). cis effects were observed in a small number of genes. PRY3, for example, a cell wall associated protein, was more strongly bound (1.4-fold) in S. paradoxus, while AMN1, a modulator of cell separation and mitotic exit, showed preferred binding (1.5-fold) to the S. cerevisiae promoter (Fig 4C and S5C). In both cases, one Ace2 binding site was absent from the promoter showing lower binding. Further, expression of the respective alleles varied in cis (within the hybrid), supporting the functionality of this gain (or loss) of binding site. We also noted reduced Ace 2 binding to the CLN3 promoter, which was associated with the loss of a well-studied Ace2 binding site[49] (S5D Fig). This binding site was previously implicated in extending G1 duration, suggesting that its loss in S. paradoxus may account for its short G1. Mutating this binding site to match the S. paradoxus allele lead to a mild but significant reduction (5\%) in G1 duration in S. cerevisiae as measured both by DNA staining and live microscopy (S5D-F Figs). Yet this cannot account, by itself, for the reduced G1 duration of S. paradoxus (50\% reduction compared to S. cerevisiae[20]).

The lower binding of Ace2 to cell-separation promoters in S. paradoxus is therefore due to variations in trans. We next asked whether such trans variations affect also the binding of Ace2's paralog, Swi5. Swi5 was bound to the same DNA motif as Ace2 in both species (S4C Fig). In contrary to Ace2, Swi5 promoters binding specificities were largely conserved between the species and within the hybrid ( $R=0.93,0.96$, respectively, Figs $4 D$ and $4 E$ top). While showing a considerable overlap with Ace 2 promoters (Fig 4F), Swi5 binding to cell separation promoters was significantly weaker than that of Ace2 (Figs 4D and 4E bottom). Therefore, the trans variations affect Ace 2 binding specifically, without affecting its paralog, Swi5.

\section{Fkh1 and Fkh2 mediate Ace2 binding to cell-separation promoters}

Fkh1 and Fkh2 were previously shown to bind Ace2-specific promoters and block their activation by Swi5[54]. We therefore hypothesized that Fkh1 and Fkh2 may regulate Ace2 activity by promoting its binding to specific promoters, including cell-separation promoters. Profiling the binding of Fkh1 and Fkh2 revealed that while these TFs bind a distinct set of promoters than Ace2 and Swi5 (Fig 4F), cell-separation genes were bound by Fkh1, and to lesser extent by Fkh2 (Figs 
$4 \mathrm{G}$ and $4 \mathrm{H}$ ). Additionally, these genes showed higher expression in S. cerevisiae than in $S$. paradoxus, which was mostly buffered in the hybrid (Fig 4G bottom). Overall, cell separation promoters that are more strongly bound by Ace 2 in S. cerevisiae, are bound also by Fkh1 and FKH2 but not by Swi5 in both species and in the hybrid.

To examine whether Fkh1 and Fkh2 recruit Ace2 to cell-separation promoters, we examined S. cerevisiae cells deleted of FKH1, FKH2 or both. Deleting either FKH1 or FKH2 had little effect on binding of Ace2 and Swi5 ( $R=0.92-0.99$, Fig $5 A$ ). Swi5 binding was also largely invariant to the deletion of both factors $(R=0.93)$, yet Ace2 binding to its top promoters was largely suppressed $(R=0.53$, Fig $5 A)$. More specifically, deletion of both FKH1 and FKH2 affected Ace2 binding primarily in promoters showing high trans variations (Fig 5B). Since these double-deletion cells are filamentous, we controlled for non-specific effects of the filamentous phenotype by overexpression of CLB2 in this background[53]. While this overexpression retrieved yeast growth, Ace2 did not regain binding to cell-separation promoters (S6B and S6C Figs). Together, these results suggest that Fkh1 and Fkh2 directly regulate the recruitment of Ace 2 to promoters, and play a role in the inter-species trans variations of Ace 2 binding.

We reasoned that the low levels of FKH1 and FKH2 in S. paradoxus could explain the reduced binding of Ace 2 to cell-separation promoters in this species. Contrasting this possibility, however, low FKH2 levels expressed using the $S$. paradoxus promoter in $S$. cerevisiae were sufficient to retrieve Ace 2 binding to FKH1/2-deleted cells (Figs 5C and S3C). Therefore, an additional layer of variations exists, whereby low levels of $\mathrm{FKH} 2$ are sufficient to promote Ace2 binding in $S$. cerevisiae but not sufficient for its recruitment in S. paradoxus. These variations could act in trans through additional signaling or other unknown factor, or could act in cis by modulating Ace2Fkh1/2 promoter interactions. Further experiments are required to test that.

\section{Discussion}

A major challenge in the study of regulatory evolution is to link variation in gene expression to differences in phenotypes. Here, we examine this by comparing budding yeast species that express largely the same set of genes and exhibit similar growth requirements, yet show major 
differences in cell-cycle dynamics, budding pattern, cell morphology, and adhesion properties when growing in the same environment. While these differences between the species encompass a wide range of cellular functions, they can be collectively explained as two distinct growth modes, yeast and filamentous, available to the individual species. This suggests that this major divergence results from the differential activation of a pre-existing differentiation program, rather than optimization of each property individually. Regulators of the yeast-to-filamentous transition are well characterized, providing a tractable model for analyzing systematically the relation between variation in gene expression and differences in phenotypes.

The transition to filamentous growth is regulated by evolutionarily conserved signaling pathways[17,57]. One of these is the MAPK cascade that activates the transcription factor, Tec1. We found that most activators in this pathway are expressed at higher levels in S. paradoxus, while inhibitors expression is higher in S. cerevisiae (Fig 5D). Variants of one such inhibitor, Ira2, was previously reported to be involved in morphological variation in wild S. paradoxus isolates [21], supporting the notion that these expression variations could have a large phenotypic effect. Expression of TEC1 itself was 4-folds higher in S. paradoxus, and this was largely due to genelinked mutation (cis effect). This, together with the ability to induce filamentous growth through TEC1 overexpression, poised Tec1 as a promising candidate for driving the phenotypic difference. However, contrasting our expectation, Tec1 is in fact dispensable for S. paradoxus filamentous growth. Examining Tec1-target genes, as defined in S. cerevisiae, provided a partial explanation for this result, as these genes accumulated cis mutations rendering their higher expression in $S$. paradoxus largely independently of Tec1. Therefore, while genes of the MAPK and its targets accumulated cis mutations that biased their expression in the direction favoring the respective phenotype, its downstream-acting TF is in fact not sufficient for explaining the difference in phenotype.

Subsequent analysis of cell-cycle regulators led to a similar conclusion; we noted that expression of the TFs FKH1 and FKH2 is lower in S. paradoxus and this reduced expression propagates downstream, affecting their targets, as well as targets of their downstream TFs, Ace 2 and Swi5 (Fig 5D). Furthermore, we found that Fkh1 and Fkh2 not only activate ACE2 and SWI5 expression, but also recruit Ace2 to cell separation genes specifically in S. cerevisiae. Yet, while S. cerevisiae cells deleted of both FKH1 and FKH2 are filamentous, expressing FKH2 using the weak $S$. paradoxus promoter was sufficient to retrieve yeast growth. 
Based on these results, we propose that the initial steps of this differential recruitment did occur through a genetic shortcut whereby a single regulator was modified to activate or repress the phenotypic switch in a new context, as often seen in experimental evolution assays[58,59]. However, once this program has been initiated, additional mutations continued to accumulate and spread throughout the regulatory network. This way, variations that bias expression in the 'wrong' direction will be eliminated by purifying selection, while those which promote the selected program will have a higher chance to persist, and will compensate for possible deleterious mutations in the initial driver. Indeed complex traits are mostly polygenic, and adaptive selection on such a trait is predicted to affect many loci[12,15]. The resulting distributed stabilization may explain the difficulty in identifying causal variations, and the prominence of both cis and trans variations that correlate with the phenotype[60].

Our model, proposing distributed stabilization of adapted complex traits, follows the conceptual framework of the omnigenic model for genetic variations[13,61] (Fig 5E). The omnigenic model explains the abundance of weak effects in genetic association studies as a consequence of the high connectivity within the transcriptional network. This connectivity expands the space of variations propagating to modulate the principle phenotypic-relevant pathway. In this framework, each individual carries a different representative of this possible pool of variations, explaining the small effect of each variant. In our case, we consider variations that have all been stabilized within the same species. The abundance of variations whose effect can influence the respective program results in a compensatory manner, which spreads the effect of each individual mutation. Therefore, each individual variation will have a small effect, leading to a distributed stabilization of all effects. Further studies are required to examine whether this model applies also in commonly studied cases such as evolutionary co-option that shape the body pattern.

\section{Materials and Methods}

\section{Strains and plasmids}

All strains used in this study and their genotypes are listed in Table S1. For timecourse experiments, Strains used were S. cerevisiae of background BY4743 (Diploid) and S. paradoxus of 
background CBS432 (Diploid). Hybrid was created by mating S. cerevisiae Mat a with S. paradoxus Mat alpha.

For ChEC-seq experiments, each transcription factor (TF) was C-terminally tagged with MNase. Yeast cells were transformed with the amplification product of MNase-Kanamycin cassette from pGZ108 plasmid, a gift from Steven Henikoff. Standard transformation using 50bp homologybased recombination was used. Hybrids were created by mating haploid TF-MNase strain, both of S. cerevisiae and S. paradoxus, with WT haploids of the other species to create reciprocal hybrids, each expressing a TF-MNase allele of either species. ChEC-seq experiments on Ace2, Swi5, Fkh1 and Fkh2 were done on haploid strains. Ace2 and Swi5 were additionally checked in diploid strains (expressing one copy of TF-MNase) to control for ploidy effects.

Gene deletions were generated by replacing each relevant gene's ORF by transformation and

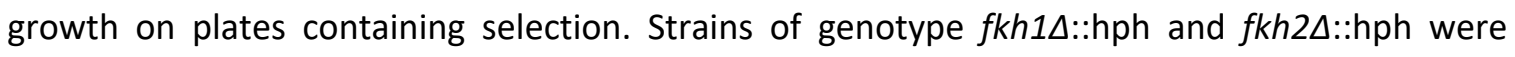
generated by amplifying the hph gene from plasmid pBS35. Double deletion of genotype $f k h 1 \Delta f k h 2 \Delta$ were generated by amplifying the LEU2 gene from plasmid pRS425 and replacing FKH2's ORF. Strains of genotype ace $2 \Delta:: K a n M X$ and swi5 $\Delta:: K a n M X$ were generated as reported at Krieger et al[62].

Swapped FKH2 strain was created in two steps: first, the FKH2's ORF of S. cerevisiae, including terminator and promoter (150bp downstream and 700bp upstream, respectively), was replaced by an amplicon of the KanMX gene from plasmid pBS7. Second, a CRISPR based method was used to induced break in the KanMX gene, while co-transforming with a genomic amplicon of FKH2 (including terminator and promoter) from S. paradoxus. Cas9 and the locus-specific 20bp gRNA were expressed using plasmid bRA89. Ligation of the gene-specific gRNA into the bRA89 plasmid was done as previously described[63].

All strains were validated by PCR and sequencing.

\section{Microscopy}

Yeast cells were grown in YPD over night at $30^{\circ} \mathrm{C}$ to stationary phase, and were inoculated to fresh medium for a few hours until reaching OD600 of 0.2. The cells were then prepared for imaging on YPD 2\% low-melt agar pads in 96-well plate. Images were taken either in an Olympus IX83 based Live-Imaging system equipped with CSU-W1 spinning disc: sCMOS digital Scientific Grade Camera 4.2 MPixel Growth or Zeiss Axio Observer Z1 inverted microscope equipped with a motorized XY 
and Z stage, external excitation and emission filter wheels (Prior), IR-based Definite Autofocus from Zeiss and a $63 \times$ oil objective. The cells were kept at $30{ }^{\circ} \mathrm{C}$. Images were taken every 5 minutes. image adjustments and labeling were performed using imageJ[64].

\section{Time-course experiments}

Yeast cells were grown in YPD over night at $30^{\circ} \mathrm{C}$ to stationary phase, and were inoculated to fresh medium to OD600 of 0.005 . When reaching an OD600 of 0.1-0.2, hydroxyurea (HU) was added to the media to a final concentration of $0.2 \mathrm{M}$ for additional 2 hours. To remove $\mathrm{HU}$ from the media, the cells were washed twice from by centrifugation (4000rpm for 1 minute) and resuspended in fresh, warm, equal-volume YPD. Then, the culture was returned to a bath orbital shaker. Cells were collected at the following time points: before $\mathrm{HU}, 5^{\prime}, 10^{\prime}, 20^{\prime}, 30^{\prime}, 60^{\prime}$ and 120 minutes in $\mathrm{HU}$, and every 5 minutes after release for $3 \mathrm{hrs}$. In total 43 time points for each strain. For RNA, samples of $1.5 \mathrm{ml}$ were taken and centrifuged for 10 seconds in 13,000 rpm, sup was removed and the pellets were immediately frozen in liquid nitrogen. For DNA staining, samples of $1.5 \mathrm{ml}$ were taken and centrifuged for 10 seconds in 13,000 rpm and resuspended in cold $70 \%$ ethanol and kept in $4^{\circ} \mathrm{C}$. This experiment was carried with two independent biological repeats for each strain.

\section{Flow Cytometry - DNA staining}

Cells were washed twice with $50 \mathrm{mM}$ Tris $-\mathrm{HCl}$ pH8, re-suspended in RNase A for 40 minutes in $37^{\circ} \mathrm{C}$, washed twice with $50 \mathrm{mM}$ Tris- $\mathrm{HCl} \mathrm{pH} 8$, and re-suspended in Proteinase $\mathrm{K}$ for 1-hour incubation at $37^{\circ} \mathrm{C}$. Then, cells were washed twice again, and re-suspended in SYBR green (1:1000) and incubated in the dark at room temperature for 1 hour. Next, cells were washed from the stain, re-suspended in 50mM Tris- $\mathrm{HCl} \mathrm{pH8}$ and sonicated in Diagenode bioruptor for 3 cycles of 10" ON and 20" OFF in low intensity. Cells were taken to FACS for analysis using BD LSRII system.

\section{RNA extraction and sequencing}

RNA was extracted using a modified protocol of nucleospin ${ }^{\circledR} 96$ RNA kit (Machery-Nagel, cat 740466.4). Specifically, cells lysis was done in a 96 deep-well plate by adding $450 \mu$ l of lysis buffer containing $1 \mathrm{M}$ sorbitol (SIGMA-ALDRICH), 100mM EDTA and $0.45 \mu \mathrm{l}$ lyticase (10IU/ $\mu \mathrm{l})$. The plate was incubated in $30^{\circ} \mathrm{C}$ for 30 minutes in order to break the cell wall, centrifuged for $10^{\prime}$ at 2500 rpm, and supernatant was removed. From this stage, extraction proceeded as in the protocol of nucleospin $^{\circledR} 96$ RNA kit, only substituting $\beta$-mercaptoethanol with DTT. cDNA was prepared from 
the RNA extracts, barcoded, and sequenced using either Illumina HiSeq 2500 or Illumina NextSeq 500.

\section{Processing and analysis of RNAseq data}

A pipeline for RNAseq data was created by Gil Hornung (INCPM,WIS). Fastq files were preprocessed by Merging them into one file, removal of reads with high A or T content, trimming of the first 3 bases (usually GGG) and trimming of Illumina adapters and bases with quality $<10$ using cutadapt.

The reads were then mapped against a dual-species reference genome of $S$. cerevisiae and $S$. paradoxus strain CBS432[65]. Genomes fasta and annotation files were downloaded from http://sss.genetics.wisc.edu/cgi-bin/s3.cgi. Mapping was performed with STAR 2.4.2a with the parameters --sjdbOverhang 60 --scoreGap -10. The alignments were divided based on the alignment scores. If for a read the highest scoring alignment is assigned to a certain genome, and is unique in that genome, then it is assigned to that genome. If there is no difference in the scores between the two genomes and the alignment is unique in the cerevisiae genome, then the alignment to the cerevisiae genome is kept and save as "indistinguishable". On average, $85 \%$ of aligned reads were mapped to either genome. Indistinguishable reads were discarded from further analysis.

Counting was performed on the TES (Transcript End Site) region of each gene. The TES region is defined as $200 \mathrm{bp}$ downstream from the end of the gene, and 500 bases upstream to the end of the gene. The reads were counted using htseq-count, with parameters --stranded yes and --mode union. The sum of all reads in each sample was normalized to be 1,000,000 and genes with expression below a threshold of $\log 2(10)$ were excluded. All further data analysis was performed in Matlab.

\section{Expression of periodic genes}

Periodic transcripts were defined by Cyclebase[39], and classified to groups based on Cyclebase expression peak time score. The top 500 genes were taken and intersected with genes that were detectable in our experiment for both species, resulting in $\sim 430$ periodic genes. For determining if a gene show periodic expression, these 430 gene were classified into 12 groups based on expression peak time, the average expression along the timecourse of each group was calculated and the pearson correlation between each gene to the average of its group was calculated. 
Significant correlations were those with corrected p-value $<0.05$ (after Benjamini Hochberg FDR procedure), and rho $>0.4$.

\section{Expression of phenotype associated genes}

Genes with positive and negative effect of filamentous growth were assembled from the Saccharomyces Genome Database[42] (SGD). Positive regulators were defined as genes that when deleted, lead to reduced filamentous growth, or when over-expressed lead to an increase in filamentous growth. Negative regulators were defined as genes that when deleted, lead to an increase in filamentous growth, or when over-expressed lead to reduced filamentous growth. Only genes that were listed under 'experiment type: classical genetics' were used.

TEC1 regulated genes were computed from Madhani et al.[24] by comparing TEC1-over expression strain to that of tec1 $\Delta$. Genes above noise level were taken and sorted by their fold change difference (Fig S2A).

\section{Gene expression of mutants}

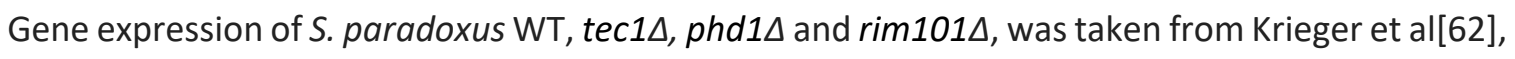
using the average expression for two biological repeats for each mutant during exponential growth in YPD.

\section{Differential expression analysis}

Differential expression analysis was carried out using DESeq2[66] on R 3.6.3. Read counts were given as input to DESeq2 with the design: " genotype", where "genotype" differentiates the sample as coming from: cerevisiae, paradoxus, hybrid-cerevisiae, hybrid-paradoxus. This design enabled differential expression analysis between species and between hybrid alleles (cis effect). Differential expression was determined via likelihood ratio test (test="LRT") focusing only on the interaction term For both designs, results went through log2 fold change shrinkage using ashr method[67].

\section{ChEC-Seq experiments}

The experiments were performed as described previously[55], with some modifications. Cultures were grown overnight to saturation in YPD media and diluted into $50 \mathrm{ml}$ of fresh YPD media to reach $\mathrm{OD}_{600}$ of $\sim 0.3$ the following morning after $\sim 10$ divisions. Cultures were pelleted at $1500 \mathrm{~g}$ and resuspended in $1 \mathrm{ml}$ Buffer $\mathrm{A}(15 \mathrm{mM}$ Tris $\mathrm{pH} 7.5,80 \mathrm{mM} \mathrm{KCl}, 0.1 \mathrm{mM}$ EGTA, $0.2 \mathrm{mM}$ 
spermine, $0.5 \mathrm{mM}$ spermidine, $1 \times$ Roche cOmplete EDTA-free mini protease inhibitors, $1 \mathrm{mM}$ PMSF), and then transferred to DNA low-bind tubes (Eppendorf 022431021). Cells were washed twice more in $500 \mu \mathrm{l}$ Buffer A, pelleted, and resuspended in $200 \mu \mathrm{l}$ Buffer A containing 0.1\% digitonin. Then, cells were transferred to an Eppendorf 96-well plate (Eppendorf 951020401) for permeabilization $\left(30^{\circ} \mathrm{C}\right.$ for $5 \mathrm{~min}$ ). $\mathrm{CaCl}_{2}$ was added to a final concentration of $2 \mathrm{mM}$ for 30 seconds for MNase activation. Next, $100 \mu$ of stop buffer (400 mM NaCl, 20 mM EDTA, 4 mM EGTA and 1\% SDS) were mixed with $100 \mu \mathrm{l}$ sample. Proteinase K (100 $\mu \mathrm{g}$, Sigma P2308) was then added, and incubated at $55^{\circ} \mathrm{C}$ for $30 \mathrm{~min}$. Nucleic acid extraction was performed as previously described[55], with some modifications in the ethanol precipitation step; Samples were precipitated (at $-80^{\circ} \mathrm{C}$ for $>1$ hour) with 2.5 volumes of cold EtOH $96 \%, 45 \mu \mathrm{g}$ Glycoblue (Thermo Fisher AM9515) and sodium acetate to a final concentration of $20 \mathrm{mM}$. DNA was centrifuged, washed with ethanol and treated with RNase A (Sigma, R4875) as previously described, followed by another round of DNA cleanup and ethanol precipitation. In order to enrich for small DNA fragments, reverse $0.8 \mathrm{X}$ SPRI clean-up was carried out. Library preparation was done as previously reported[68], except for the clean-up steps, which were performed using phenol-chloroform followed by ethanol precipitation as described above (instead of S400 columns). 1X SPRI was carried out on ChEC amplified libraries, which were then pooled and cleaned from adaptors dimers (150bp) if needed. Libraries were sequenced on Illumina NextSeq500 for paired end sequencing ( 50 bps for read 1 and 15 or 25 bps for read 2).

Library construction for of ChEC-seq of Hybrid strain of FKH1-MNase and FKH2-MNase was slightly modified and phenol-chloroform clean-ups were replaced by SPRI-isopropanol clean-ups[69].

All ChEC-seq experiments were repeated at least twice for each strain.

\section{ChEC-Seq processing and analysis}

Reads were aligned using Bowtie2[70] (parameters: -best $-\mathrm{m} 1$ ) to a dual-species reference genome of S. cerevisiae R64 and S. paradoxus CBS432[71]. ChEC-Seq tracks, representing the enrichment of each $\mathrm{TF}$, were calculated by adding +1 to each genomic location corresponding to the first nucleotide in a forward read, or the 50th position corresponding a reverse read. The signal was normalized to a total of 10 million reads, to control for sequencing depth. The median signal across repeats for each strain was taken for further analysis. For promoter analysis, 
promoters were defined only for genes with an annotated transcription start site (TSS)[72]. The length of each promoter was defined as 700 bps upstream to the transcription start site (TSS). The signal across each promoter was summed to calculate overall promoter binding for each sample.

\section{Acknowledgments}

We thank all memebers of the Barkai lab. We thank Gil Hornung (INCPM, Weizmann Institute of Science) for constructing the RNA-seq pipeline. We thank Sagie Brodsky for both technical and computational help in ChEC-seq experiments and data analysis. We thank Dana Bar-Zvi, Tamar Jana, Gilad Yaakov and Miri Carmi for additional technical help and fruitful discussions.

\section{References}

1. Kimura M. Evolutionary rate at the molecular level. Nature. 1968;217(5129):624-6.

2. Carroll SB. Endless forms: the evolution of gene regulation and morphological diversity. Cell [Internet]. 2000 Jun 9 [cited 2018 Mar 25];101(6):577-80. Available from: http://www.ncbi.nlm.nih.gov/pubmed/10892643

3. Carroll SB. Evo-Devo and an Expanding Evolutionary Synthesis: A Genetic Theory of Morphological Evolution. Vol. 134, Cell. Cell Press; 2008. p. 25-36.

4. King MC, Wilson AC. Evolution at two levels in humans and chimpanzees. Science [Internet]. 1975 Apr 11 [cited 2018 Apr 9];188(4184):107-16. Available from: http://www.ncbi.nlm.nih.gov/pubmed/1090005

5. Carroll SB. Homeotic genes and the evolution of arthropods and chordates. Nature. 1995 Aug 10;376(6540):479-85.

6. Jeong S, Rokas A, Carroll SB. Regulation of Body Pigmentation by the Abdominal-B Hox Protein and Its Gain and Loss in Drosophila Evolution. Cell. 2006 Jun 30;125(7):1387-99.

7. Gomez C, Özbudak EM, Wunderlich J, Baumann D, Lewis J, Pourquié O. Control of segment number in vertebrate embryos. Nature. 2008 Jul 17;454(7202):335-9.

8. Brakefield PM, Gates J, Keys D, Kesbeke F, Wijngaarden PJ, Monteiro A, et al. 
Development plasticity and evolution of butterfly eyespot patterns. Vol. 384, Nature. Nature Publishing Group; 1996. p. 236-42.

9. Martin A, Papa R, Nadeau NJ, Hill RI, Counterman BA, Halder G, et al. Diversification of complex butterfly wing patterns by repeated regulatory evolution of a Wnt ligand. Proc Natl Acad Sci U S A. 2012 Jul 31;109(31):12632-7.

10. Keys DN, Lewis DL, Selegue JE, Pearson BJ, Goodrich L V., Johnson RL, et al. Recruitment of a hedgehog regulatory circuit in butterfly eyespot evolution. Science (80- ). 1999;283(5401):532-4.

11. True JR, Carroll SB. Gene Co-Option in Physiological and Morphological Evolution. Annu Rev Cell Dev Biol. 2002 Nov;18(1):53-80.

12. Sella G, Barton NH. Thinking About the Evolution of Complex Traits in the Era of GenomeWide Association Studies. Annu Rev Genomics Hum Genet [Internet]. 2019 Aug 31 [cited 2020 Apr 19];20(1):461-93. Available from:

https://www.annualreviews.org/doi/10.1146/annurev-genom-083115-022316

13. Liu X, Li YI, Pritchard JK. Trans Effects on Gene Expression Can Drive Omnigenic Inheritance. Cell. 2019 May 2;177(4):1022-1034.e6.

14. Liu Y, Ramos-Womack M, Han C, Reilly P, Brackett KLR, Rogers W, et al. Changes throughout a Genetic Network Mask the Contribution of Hox Gene Evolution. Curr Biol. 2019 Jul 8;29(13):2157-2166.e6.

15. Monteiro A, Podlaha O. Wings, Horns, and Butterfly Eyespots: How Do Complex Traits Evolve? PLoS Biol [Internet]. 2009 Feb 24 [cited 2020 May 21];7(2):e1000037. Available from: https://dx.plos.org/10.1371/journal.pbio.1000037

16. Rice G, Rebeiz M. Evolution: How Many Phenotypes Do Regulatory Mutations Affect? Vol. 29, Current Biology. Cell Press; 2019. p. R21-3.

17. Cullen PJ, Sprague GF. The regulation of filamentous growth in yeast. Genetics. 2012 Jan $1 ; 190(1): 23-49$.

18. Palecek SP, Parikh AS, Kron SJ. Sensing, signalling and integrating physical processes during Saccharomyces cerevisiae invasive and filamentous growth. Vol. 148, Microbiology. Society for General Microbiology; 2002. p. 893-907. 
19. Madden K, Snyder M. CELL POLARITY AND MORPHOGENESIS IN BUDDING YEAST [Internet]. Vol. 52, Annu. Rev. Microbiol. 1998 [cited 2020 Apr 13]. Available from: www.annualreviews.org

20. Herbst R, Bar-Zvi D, Reikhav S, Soifer I, Breker M, Jona G, et al. Heterosis as a consequence of regulatory incompatibility. BMC Biol. 2017 Jul 19;297(5580):395-400.

21. Roop JI, Brem RB. Rare variants in hypermutable genes underlie common morphology and growth traits in wild Saccharomyces paradoxus. Genetics [Internet]. 2013 Oct [cited 2017 Apr 24];195(2):513-25. Available from: http://www.ncbi.nlm.nih.gov/pubmed/23934881

22. Ryan O, Shapiro RS, Kurat CF, Mayhew D, Baryshnikova A, Chin B, et al. Global gene deletion analysis exploring yeast filamentous growth. Science (80- ). 2012 Sep $14 ; 337(6100): 1352-6$.

23. Jin R, Dobry CJ, McCown PJ, Kumar A. Large-scale analysis of yeast filamentous growth by systematic gene disruption and overexpression. Mol Biol Cell. 2008 Jan;19(1):284-96.

24. Madhani HD, Galitski T, Lander ES, Fink GR. Effectors of a developmental mitogenactivated protein kinase cascade revealed by expression signatures of signaling mutants. Proc Natl Acad Sci U S A. 1999 Oct 26;96(22):12530-5.

25. Gimeno CJ, Styles CA, Fink GR. Unipolar Cell Divisions in the Yeast S. cerevisiae Lead to Filamentous Growth: Regulation by Starvation and RAS. Vol. 66, Cell. 1992.

26. Prinz S, Avila-Campillo I, Aldridge C, Srinivasan A, Dimitrov K, Siegel AF, et al. Control of yeast filamentous-form growth by modules in an integrated molecular network [Internet]. Vol. 14, Genome Research. 2004 [cited 2020 Apr 13]. p. 380-90. Available from: http://www.ncbi.nlm.nih.gov/pubmed/14993204

27. Shively CA, Eckwahl MJ, Dobry CJ, Mellacheruvu D, Nesvizhskii A, Kumar A. Genetic networks inducing invasive growth in Saccharomyces cerevisiae identified through systematic genome-wide overexpression. Genetics. 2013;193(4):1297-310.

28. Cromie GA, Tan Z, Hays M, Sirr A, Jeffery EW, Dudley AM. Transcriptional profiling of biofilm regulators identified by an overexpression screen in Saccharomyces cerevisiae. G3 Genes, Genomes, Genet. 2017;7(8):2845-54.

29. Wittkopp PJ, Haerum BK, Clark AG. volutionary changes in cis and trans gene regulation. 
Nature [Internet]. 2004 Jul 1 [cited 2018 Mar 25];430(6995):85-8. Available from: http://www.ncbi.nlm.nih.gov/pubmed/15229602

30. Landry CR, Wittkopp PJ, Taubes CH, Ranz JM, Clark AG, Hartl DL. Compensatory cis-trans evolution and the dysregulation of gene expression in interspecific hybrids of drosophila. Genetics. 2005 Dec 1;171(4):1813-22.

31. McManus CJ, Coolon JD, Duff MO, Eipper-Mains J, Graveley BR, Wittkopp PJ. Regulatory divergence in Drosophila revealed by mRNA-seq. Genome Res [Internet]. 2010 Jun 1 [cited 2018 Mar 26];20(6):816-25. Available from: http://www.ncbi.nlm.nih.gov/pubmed/20354124

32. Tirosh I, Reikhav S, Levy AA, Barkai N. A yeast hybrid provides insight into the evolution of gene expression regulation. Science [Internet]. 2009 May 1 [cited 2017 Mar 12];324(5927):659-62. Available from: http://www.ncbi.nlm.nih.gov/pubmed/19407207

33. McManus CJ, May GE, Spealman P, Shteyman A. Ribosome profiling reveals posttranscriptional buffering of divergent gene expression in yeast. Genome Res [Internet]. 2014 Mar 1 [cited 2017 Feb 9];24(3):422-30. Available from: http://www.ncbi.nlm.nih.gov/pubmed/24318730

34. Goncalves A, Leigh-Brown S, Thybert D, Stefflova K, Turro E, Flicek P, et al. Extensive compensatory cis-trans regulation in the evolution of mouse gene expression. Genome Res. 2012 Dec 1;22(12):2376-84.

35. Albert FW, Muzzey D, Weissman JS, Kruglyak L. Genetic Influences on Translation in Yeast. Brem RB, editor. PLoS Genet [Internet]. 2014 Oct 23 [cited 2020 May 3];10(10):e1004692. Available from: https://dx.plos.org/10.1371/journal.pgen.1004692

36. Shi X, Ng DWK, Zhang C, Comai L, Ye W, Jeffrey Chen Z. Cis- and trans-regulatory divergence between progenitor species determines gene-expression novelty in Arabidopsis allopolyploids. Nat Commun. 2012 Jul 17;3(1):1-9.

37. Artieri CG, Naor A, Turgeman-Grott I, Zhou Y, York R, Gophna U, et al. Cis -regulatory evolution in prokaryotes revealed by interspecific archaeal hybrids. Sci Rep. 2017 Dec $1 ; 7(1): 1-8$.

38. Combs PA, Krupp JJ, Khosla NM, Bua D, Petrov DA, Levine JD, et al. Tissue-Specific cisRegulatory Divergence Implicates eloF in Inhibiting Interspecies Mating in Drosophila. 
Curr Biol. 2018 Dec 17;28(24):3969-3975.e3.

39. Santos A, Wernersson R, Jensen L. Cyclebase 3.0: a multi-organism database on cellcycle regulation and phenotypes. Nucleic Acids Res [Internet]. 2015 Jan 28 [cited 2018 Mar 25];43(D1):D1140-4. Available from: http://academic.oup.com/nar/article/43/D1/D1140/2437426/Cyclebase-30-amultiorganism-database-on-cellcycle

40. Granovskaia M V., Jensen LJ, Ritchie ME, Toedling J, Ning Y, Bork P, et al. High-resolution transcription atlas of the mitotic cell cycle in budding yeast. Genome Biol. 2010 Mar $1 ; 11(3)$.

41. Spellman PT, Sherlock G, Zhang MQ, lyer VR, Anders K, Eisen MB, et al. Comprehensive identification of cell cycle-regulated genes of the yeast Saccharomyces cerevisiae by microarray hybridization. Mol Biol Cell [Internet]. 1998 Dec 1 [cited 2018 Mar 20];9(12):3273-97. Available from: http://www.ncbi.nlm.nih.gov/pubmed/9843569

42. Chervitz SA, Hester ET, Ball CA, Dolinski K, Dwight SS, Harris MA, et al. Using the Saccharomyces Genome Database (SGD) for analysis of protein similarities and structure [Internet]. Vol. 27, Nucleic Acids Research. 1999 [cited 2020 Apr 13]. Available from: www.mdli.com

43. Gavrias V, Andrianopoulos A, Gimeno CJ, Timberlake WE. Saccharomyces cerevisiae TEC1 is required for pseudohyphal growth. Mol Microbiol [Internet]. 1996 Mar [cited $2020 \mathrm{Apr}$ 13];19(6):1255-63. Available from: http://doi.wiley.com/10.1111/j.1365-

2958.1996.tb02470.x

44. Lamb TM, Mitchell AP. The Transcription Factor Rim101p Governs Ion Tolerance and Cell Differentiation by Direct Repression of the Regulatory Genes NRG1 and SMP1 in Saccharomyces cerevisiae. Mol Cell Biol. 2003 Jan 15;23(2):677-86.

45. Malcher M, Schladebeck S, Mösch HU. The Yak1 protein kinase lies at the center of a regulatory cascade affecting adhesive growth and stress resistance in Saccharomyces cerevisiae. Genetics. 2011 Mar;187(3):717-30.

46. Zhu G, Spellman PT, Volpe T, Brown PO, Botstein D, Davis TN, et al. Two yeast forkhead genes regulate the cell cycle and pseudohyphal growth. Nature [Internet]. 2000 Jul [cited 2019 Apr 3];406(6791):90-4. Available from: http://www.nature.com/articles/35017581 
47. Morgan DO. The Cell Cycle: Principles of Control. New Science Press; 2007.

48. Sopko R, Huang D, Preston N, Chua G, Papp B, Kafadar K, et al. Mapping pathways and phenotypes by systematic gene overexpression. Mol Cell. 2006 Feb 3;21(3):319-30.

49. Di Talia S, Wang H, Skotheim JM, Rosebrock AP, Futcher B, Cross FR. Daughter-Specific Transcription Factors Regulate Cell Size Control in Budding Yeast. Kellogg DR, editor. PLoS Biol [Internet]. 2009 Oct 20 [cited 2017 Oct 17];7(10):e1000221. Available from: http://journals.plos.org/plosbiology/article/file?id=10.1371/journal.pbio.1000221\&type= printable

50. Weiss EL. Mitotic Exit and Separation of Mother and Daughter Cells. Genetics [Internet]. 2012 Apr 1 [cited 2017 Nov 9];190(4):1197-224. Available from: http://www.ncbi.nlm.nih.gov/pubmed/22491889

51. King L, Butler G. Ace2p, a regulator of CTS1 (chitinase) expression, affects pseudohyphal production in Saccharomyces cerevisiae. Curr Genet [Internet]. 1998 Sep [cited 2020 Apr 15];34(3):183-91. Available from: http://www.ncbi.nlm.nih.gov/pubmed/9745020

52. Laabs TL, Markwardt DD, Slattery MG, Newcomb LL, Stillman DJ, Heideman W, et al. ACE2 is required for daughter cell-specific $\mathrm{G} 1$ delay in Saccharomyces cerevisiae. [cited 2017 Sep 27]; Available from: http://www.pnas.org/content/100/18/10275.full.pdf

53. Hollenhorst PC, Bose ME, Mielke MR, Müller U, Fox CA. Forkhead genes in transcriptional silencing, cell morphology and the cell cycle. Overlapping and distinct functions for FKH1 and FKH2 in Saccharomyces cerevisiae. Genetics [Internet]. 2000 Apr [cited 2019 Dec 3];154(4):1533-48. Available from: http://www.ncbi.nlm.nih.gov/pubmed/10747051

54. Voth WP, Yu Y, Takahata S, Kretschmann KL, Lieb JD, Parker RL, et al. Forkhead proteins control the outcome of transcription factor binding by antiactivation. EMBO J [Internet]. 2007 [cited 2017 Nov 9];26:4324-34. Available from: https://www.ncbi.nlm.nih.gov/pmc/articles/PMC2034669/pdf/7601859a.pdf

55. Zentner GE, Kasinathan S, Xin B, Rohs R, Henikoff S. ChEC-seq kinetics discriminates transcription factor binding sites by DNA sequence and shape in vivo. Nat Commun [Internet]. 2015 Oct 22 [cited 2018 Jan 11];6:8733. Available from: http://www.nature.com/doifinder/10.1038/ncomms9733

56. de Boer CG, Hughes TR. YeTFaSCo: a database of evaluated yeast transcription factor 
sequence specificities. Nucleic Acids Res [Internet]. 2011 Nov 17;40(D1):D169-79. Available from: https://doi.org/10.1093/nar/gkr993

57. Kiss $E$, Hegedüs $B$, Virágh $M$, Varga $T$, Merényi Z, Kószó $T$, et al. Comparative genomics reveals the origin of fungal hyphae and multicellularity. Nat Commun. 2019 Dec $1 ; 10(1): 1-13$.

58. Hope EA, Amorosi CJ, Miller AW, Dang K, Heil CS, Dunham MJ. Experimental evolution reveals favored adaptive routes to cell aggregation in yeast. Genetics. 2017 Jun 1;206(2):1153-67.

59. Ratcliff WC, Fankhauser JD, Rogers DW, Greig D, Travisano M. Origins of multicellular evolvability in snowflake yeast. Nat Commun. 2015 Jan 20;6(1):1-9.

60. Signor SA, Nuzhdin S V. The evolution of gene expression in cis and trans HHS Public Access. Trends Genet. 2018;34(7):532-44.

61. Boyle EA, Li YI, Pritchard JK. An expanded view of complex traits: from polygenic to omnigenic.

62. Krieger G, Lupo O, Levy AA, Barkai N. Independent evolution of transcript abundance and gene regulatory dynamics. bioRxiv [Internet]. 2020 Jan 23 [cited 2020 Apr 16];2020.01.22.915033. Available from: http://dx.doi.org/10.1101/2020.01.22.915033

63. DiCarlo JE, Norville JE, Mali P, Rios X, Aach J, Church GM. Genome engineering in Saccharomyces cerevisiae using CRISPR-Cas systems. Nucleic Acids Res [Internet]. 2013 Mar 4;41(7):4336-43. Available from: https://doi.org/10.1093/nar/gkt135

64. Abràmoff MD, Magalhães PJ, Ram SJ. Image Processing with ImageJ Second Edition [Internet]. Vol. 11, Biophotonics International. Laurin Publishing; 2004 [cited 2020 Apr 16]. 36-42 p. Available from: https://imagescience.org/meijering/publications/download/bio2004.pdf

65. Scannell DR, Zill OA, Rokas A, Payen C, Dunham MJ, Eisen MB, et al. The Awesome Power of Yeast Evolutionary Genetics: New Genome Sequences and Strain Resources for the Saccharomyces sensu stricto Genus. G3 (Bethesda) [Internet]. 2011 Jun 1 [cited 2017 Feb 8];1(1):11-25. Available from: http://www.ncbi.nlm.nih.gov/pubmed/22384314

66. Love MI, Huber W, Anders S. Moderated estimation of fold change and dispersion for RNA-seq data with DESeq2. Genome Biol [Internet]. 2014 Dec 5 [cited 2020 Apr 
21];15(12):550. Available from:

http://genomebiology.biomedcentral.com/articles/10.1186/s13059-014-0550-8

67. Stephens M. False discovery rates: a new deal. Biostatistics [Internet]. 2016 Oct 17;18(2):275-94. Available from: https://doi.org/10.1093/biostatistics/kxw041

68. Henikoff JG, Belsky JA, Krassovsky K, MacAlpine DM, Henikoff S. Epigenome characterization at single base-pair resolution. Proc Natl Acad Sci U S A. 2011 Nov 8;108(45):18318-23.

69. Fishman A, Light D, Lamm AT. QsRNA-seq: A method for high-throughput profiling and quantifying small RNAs. Genome Biol [Internet]. 2018 Aug 14 [cited 2020 Apr 20];19(1):113. Available from: https://genomebiology.biomedcentral.com/articles/10.1186/s13059-018-1495-0

70. Langmead B, Salzberg SL. Fast gapped-read alignment with Bowtie 2. Nat Methods. 2012 Apr;9(4):357-9.

71. Yue JX, Li J, Aigrain L, Hallin J, Persson K, Oliver K, et al. Contrasting evolutionary genome dynamics between domesticated and wild yeasts. Nat Genet. 2017 Jun 1;49(6):913-24.

72. David L, Huber W, Granovskaia M, Toedling J, Palm CJ, Bofkin L, et al. A high-resolution map of transcription in the yeast genome. Proc Natl Acad Sci U S A. 2006 Apr 4;103(14):5320-5.

73. Maclsaac KD, Wang T, Gordon DB, Gifford DK, Stormo GD, Fraenkel E. An improved map of conserved regulatory sites for Saccharomyces cerevisiae. BMC Bioinformatics [Internet]. 2006 Mar 7 [cited 2017 Oct 4];7(1):113. Available from: http://bmcbioinformatics.biomedcentral.com/articles/10.1186/1471-2105-7-113

\section{Figures}




\section{Figure1}

A

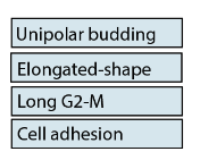

Yeast-form growth

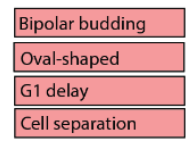

Cell separation

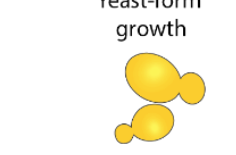

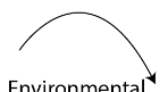

Environmental
$-\quad$ cues

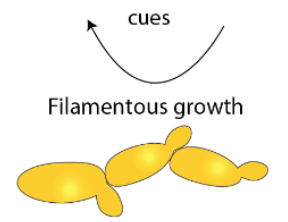

B
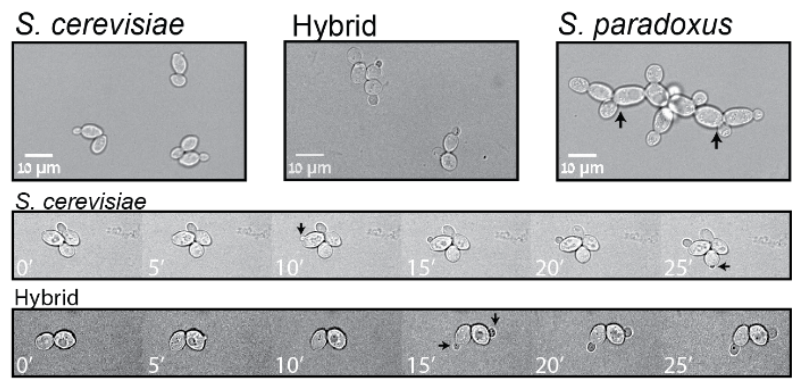

S. paradoxus
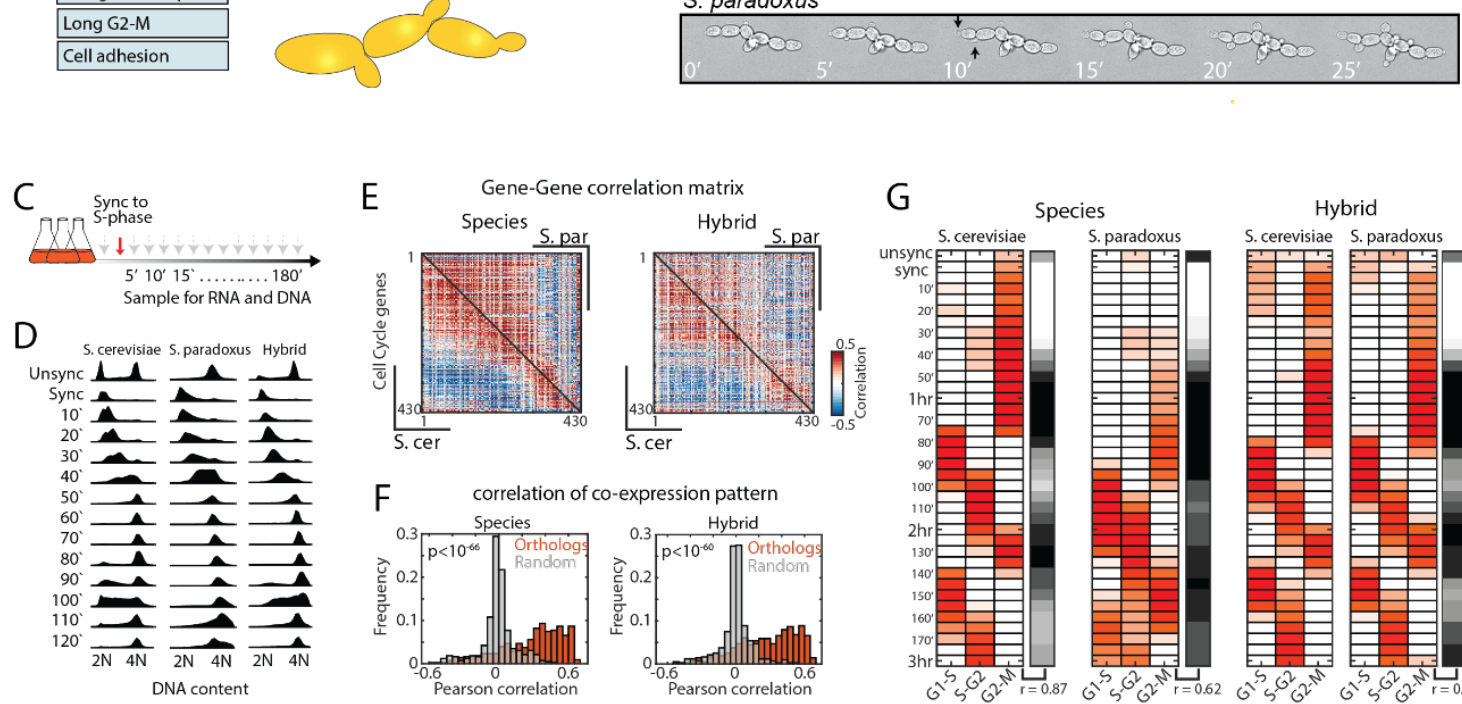

$E$

Gene-Gene correlation matrix

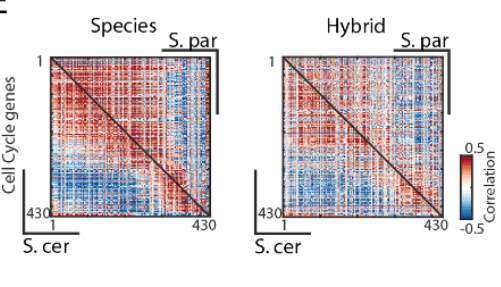

G

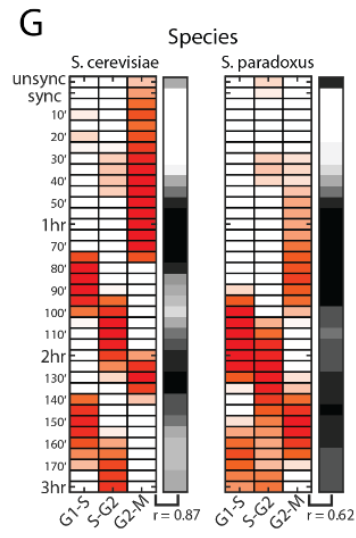

Hybrid

S. cerevisiae S. paradoxus

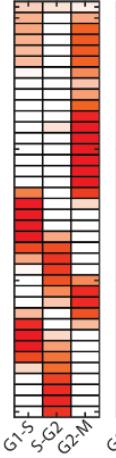

FC(log2)
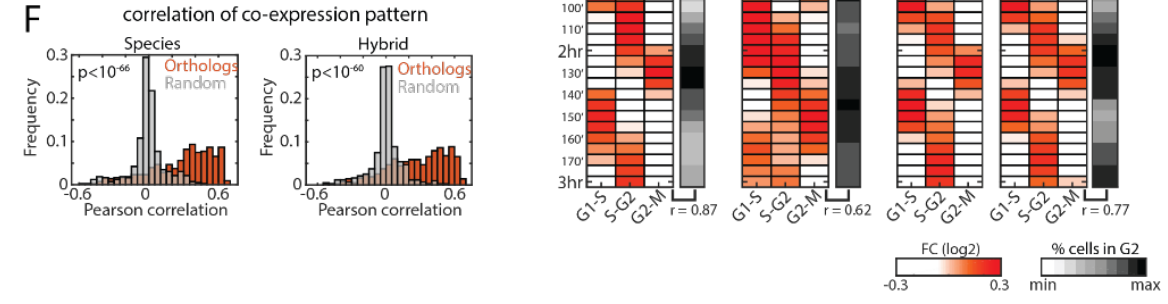

Fig 1. Trans-dominant expression program follows the growth pattern (A) A scheme of the two types of growth in budding yeast. Budding yeast can undergo a dimorphic shift upon environmental cues which involves modifying different processes in cell cycle and morphology. (B) Microscopy images of S. cerevisiae, S. paradoxus and their hybrid grown on YPD. S. cerevisiae grows as yeast while S. paradoxus grows in filaments. The hybrid grows as yeast, while exhibiting short daughter-delay. Note cell elongation, unipolar budding and synchronized mother-daughter budding in S. paradoxus. Black arrows point at new buds. (C) Experimental layout: Cells were synchronized with HU, washed and sampled every 5 minutes for three hours. (D) Synchronized progression was monitored by measuring the DNA content using flow-cytometry (full profiles are shown in Fig S1A) (E) Co-expression of cycling genes is conserved between species. Shown are gene-gene correlation matrices (Pearson) of periodic genes ordered by their expression peak 
along the cell cycle, S. cerevisiae is shown in the lower triangle and S. paradoxus in the top triangle. (F) Histograms showing the distributions of co-expression correlation coefficients between orthologous genes and between random pairs (indicated p-value of a two-samples t-test). (G) Periodicity of gene expression is synchronized with cell-cycle transitions. Shown are the average fold changes of periodic genes (relative to median) classified to three groups based on expression time. Black panel indicated percent of cells with 4N DNA content. Correlation values in bottom indicate the Pearson correlation coefficient between expression of the G2-M module to percent cells with 4N DNA content.

A

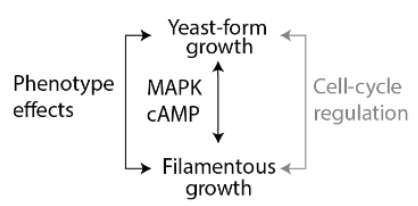

C

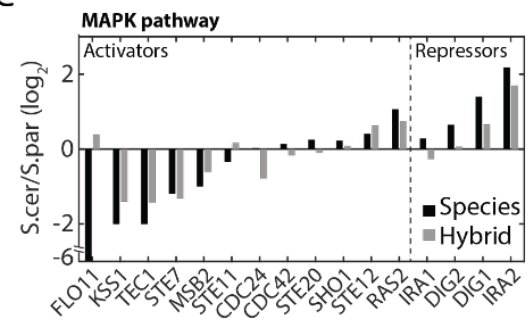

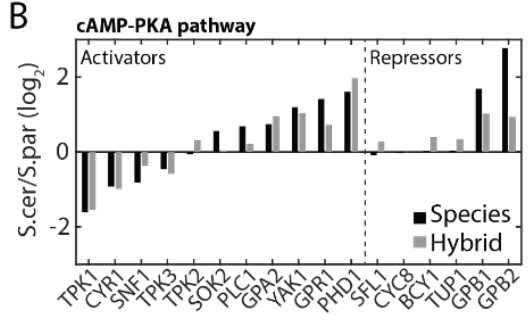

D

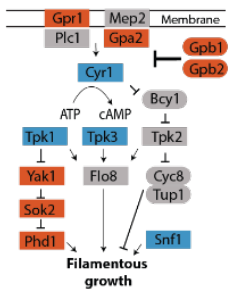

$E$

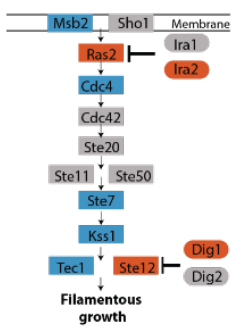

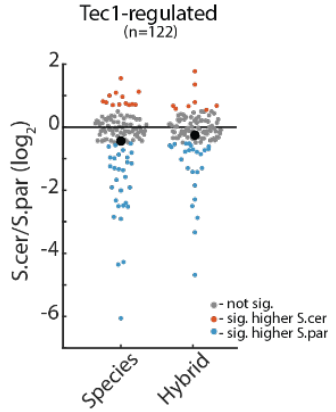

S. paradoxus WT

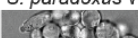

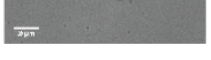

S. paradoxus tec1 $1 \Delta / \Delta$

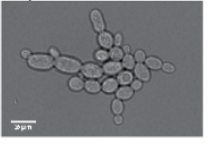

Fig 2. Filamentous regulators accumulated cis-effects that bias expression towards the selected growth program (A) a scheme representing different regulatory layers that affect the yeast-tofilamentous growth switch. (B-C) Genes in the main filamentous signaling pathways CAMP-PKA (B) and MAPK (C) show differential expression in cis and in trans. Left: Shown are $\log _{2}$ fold change $(F C)$ values of genes involved the indicated pathways. Right: Schemes representing the respected signaling cascades. Colors are given for genes showing significant FC difference between hybrid alleles (cis), as indicated in legend. (D) cis-regulation of Tec1-dependent genes. Shown are FC distributions of Tec1-regulated genes (Fig S2B). Red and blue dots indicate significantly higher expression in S. cerevisiae and S. paradoxus, respectively. Black dot marks the mean (E) Phenotypes of diploid S. paradoxus WT and tec1 $\Delta / \Delta$, growing in rich conditions (YPD). 
A

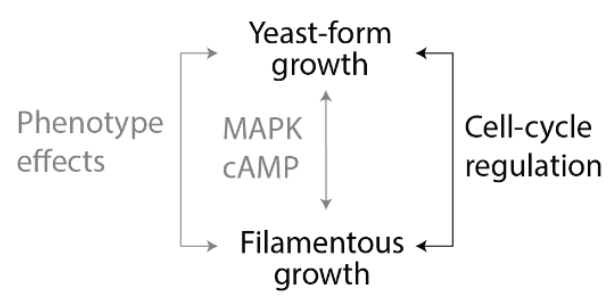

C
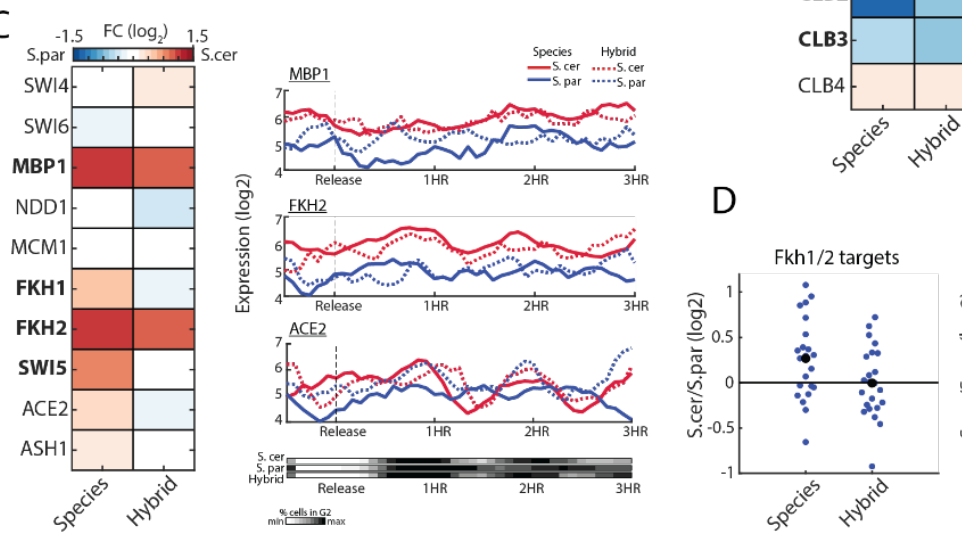

B
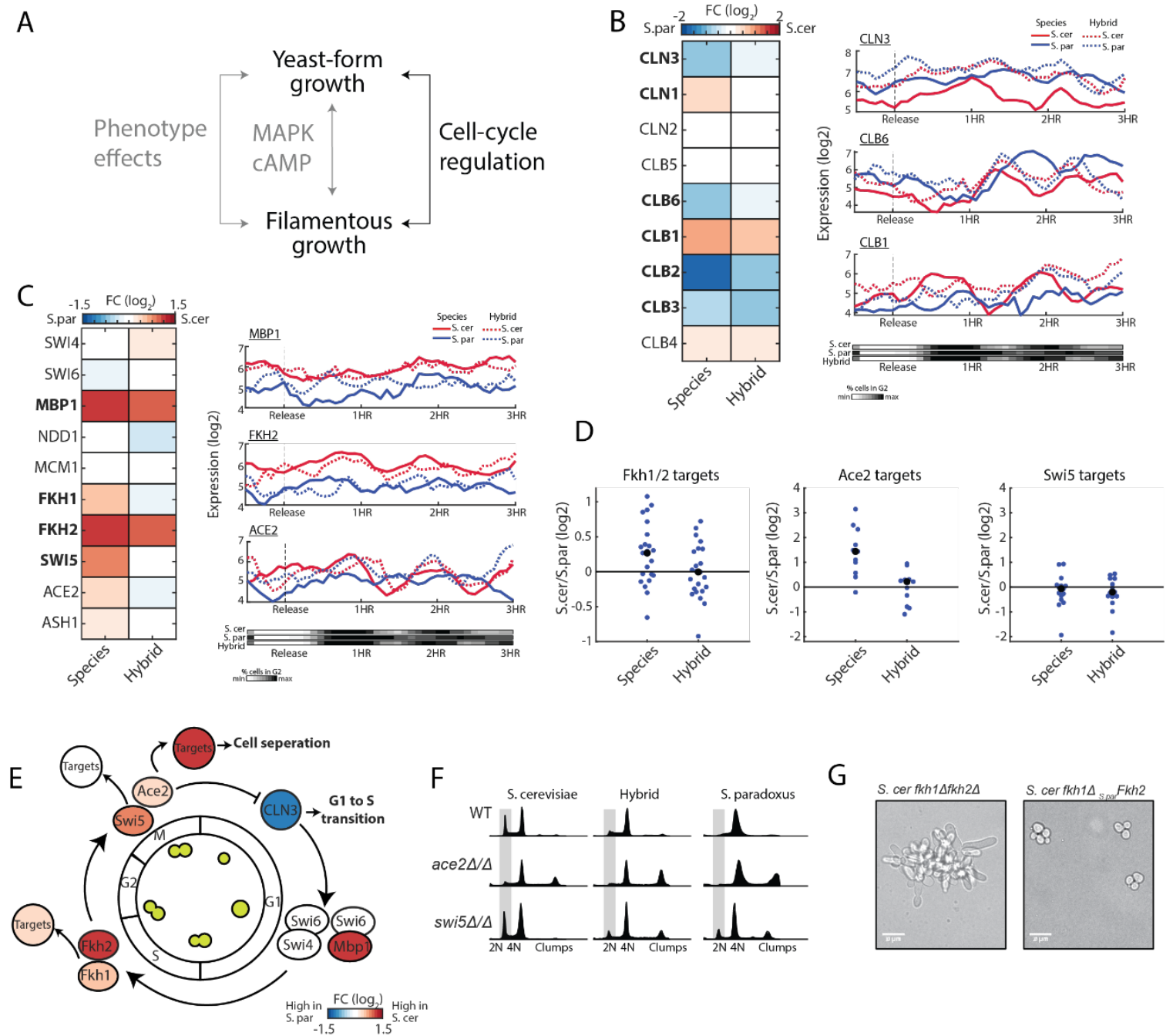

$\mathrm{F}$

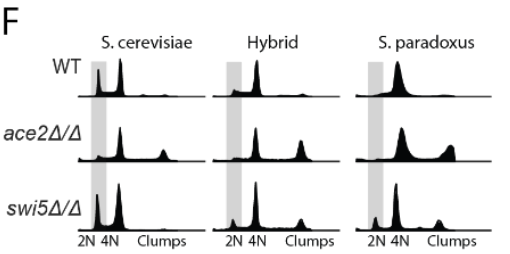

G
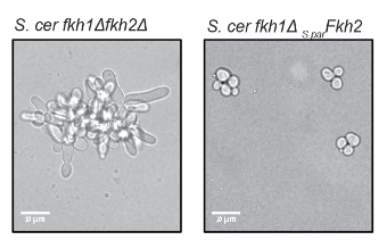

Fig 3. Cell-cycle regulators accumulated cis-effects that bias expression towards the selected

growth program (A) A scheme representing different regulatory layers that affect the yeast-tofilamentous growth switch. (B) Expression of cyclins. Left: Examples of CLN3 (early G1), CLB6 (early S) and CLB1(G2) along the cell cycle, in synchrony with changes in DNA content (bottom panel). Right: $\log _{2} \mathrm{FC}$ expression differences. Genes ordered by their expression time along the cell cycle. Genes in bold have significant differential expression between species. (C) Expression of cell cycle transcription factors (TF), same as in (A). (D) $\log _{2} \mathrm{FC}$ in Fkh1/2, Ace2 and Swi5's targets. Number indicates number of genes; Black dots indicate the group's mean. (E) Divergence in cell cycle regulators. A scheme of cell cycle regulation, color indicates inter-species expression differences. (F) DNA contents profiles of WT, ace $2 \Delta / \Delta$ and $s w i 5 \Delta / \Delta$, in both species and hybrid, taken during exponential growth in YPD. Grey area marks cells with 2N DNA content (in G1 phase). (G) 
Phenotype of S. cerevisiae $f k h 1 \Delta f k h 2 \Delta$ (left) and S. cerevisiae $f k h 1 \Delta$ expressing S. paradoxus FKH2 (right), growing in rich conditions (YPD).

Figure 4

A
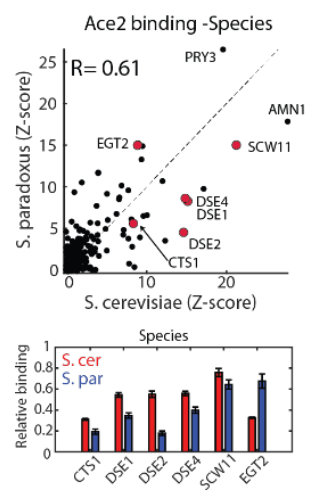

D
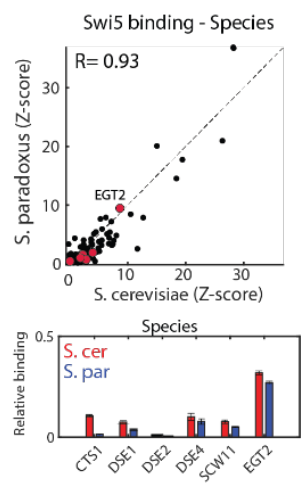

B Ace2 binding - Hybrid

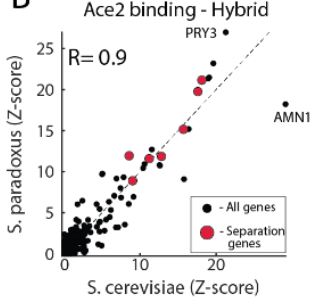

Hybrid

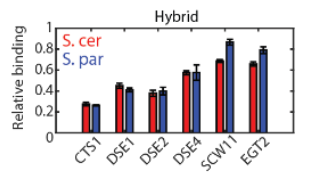

E Swi5 binding - Hybrid
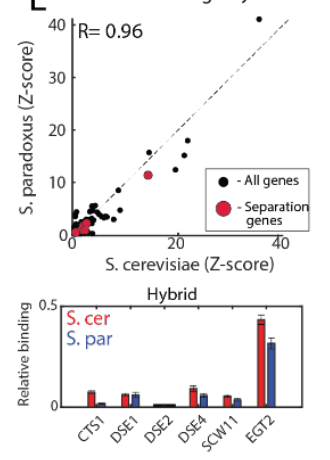

C
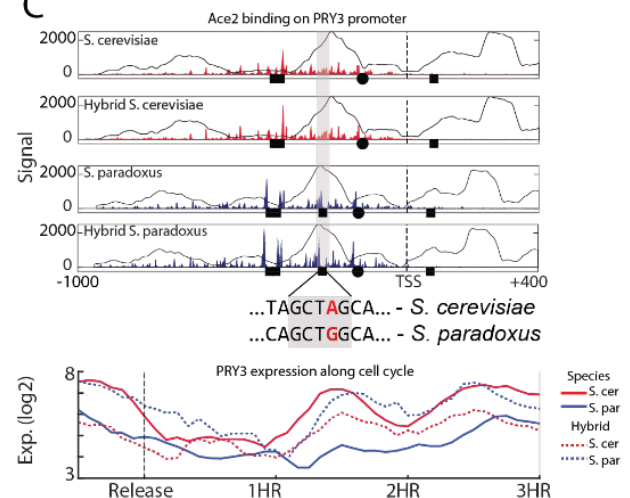

$\mathrm{F}$

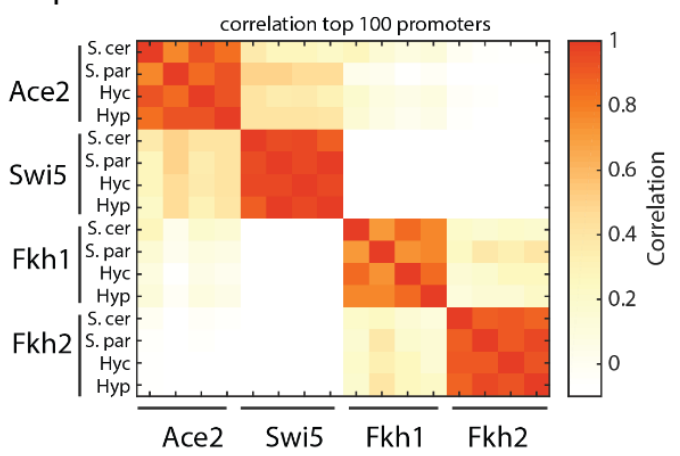

G Swi5 top promoters
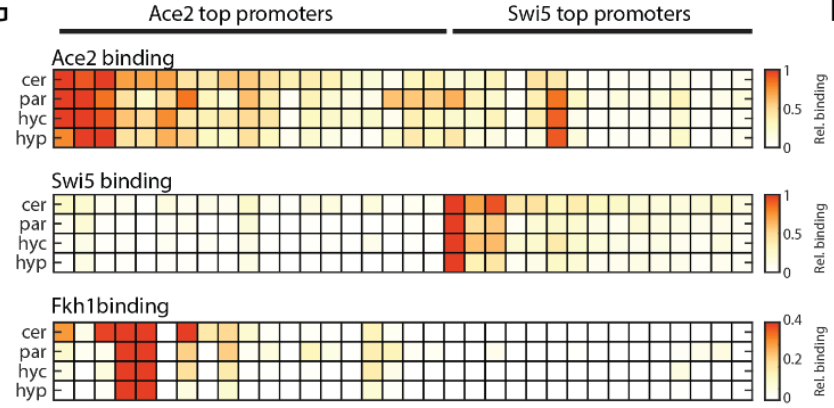

Fkh2 binding

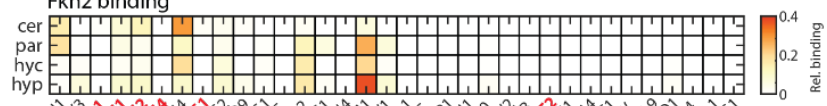

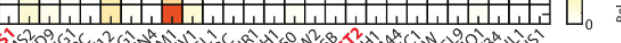

م.8,

Species Expression (S. cer/s. par)

Species
Hybrid

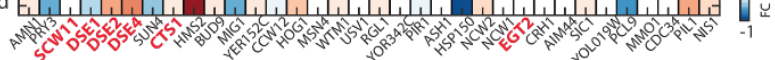

$\mathrm{H}$

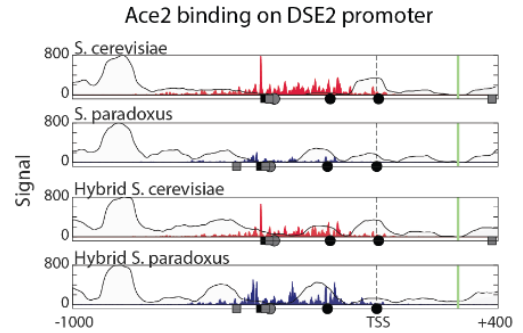

Fkh1 binding on DSE2 promoter

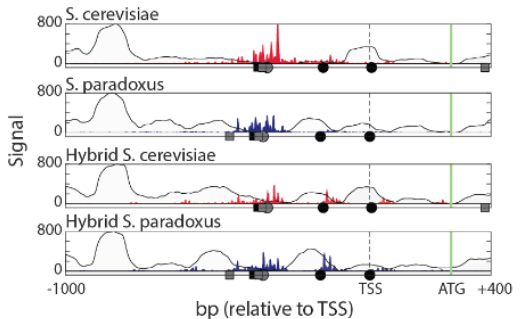

Fig 4. Cis and trans regulatory variations affect ACE2 binding (A) ChEC-seq binding profiles of Ace2 between species. Top: plotted is Ace2 sum of signal on each promoter after Z-score 
normalization. Pearson correlation values of top 100 promoters are shown, genes involved in cell separation are marked in red. Bottom: Relative Ace2 binding on cell separation genes. (B) ChECseq binding profiles of Ace2 within the hybrid, Same as in (A). (C) Ace2 differentially binds PRY3 promoter. Top: Ace2 binding on PRY3 promoter, black circles and squares represent Ace2 binding motif (CCAGC) on + and - strand, respectively. Background line represents nucleosome occupancy. Dashed line represent transcription start site (TSS), x-ticks are bp relative to TSS. Middle: Sequence changes leading to loss or gain of Ace2 motif are shown. Bottom: PRY3 expression along the cell cycle, note the cis effect between hybrid alleles. (D-E) ChEC-seq binding profiles of Swi5 between species and within hybrid, same as in (A-B). (F) Pearson correlation matrix of top 100 promoters of Ace2, Swi5, Fkh1 and Fkh2. Hyc and Hyp refer to Hybrid S. cerevisiae genome and S. paradoxus genome, respectively. Note that paralogs show considerable correlations, Fkh1 show low positive correlation with S. cerevisiae Ace2. (G) Binding to top Ace2 and Swi5 promoters. Top: Shown are relative sum of signal on promoters for each factor in the species and hybrid. Note overlap of Fkh1 and Fkh2 with Ace2 promoters. Note that different color scale for Fkh1 and Fkh2 is shown. Bottom: Differences in expression levels. Note, cell separation genes (marked in red) show higher expression in S. cerevisiae, which is mostly lost in the hybrid (trans effect). (H) Binding of Ace2 and Fkh1 on the cell separation gene DSE2. Circles and squares represent Ace2 binding motif (CCAGC, black), and Fkh1 binding motif (GTAAACA, grey). Note trans effects both in Ace2 and in Fkh1. 

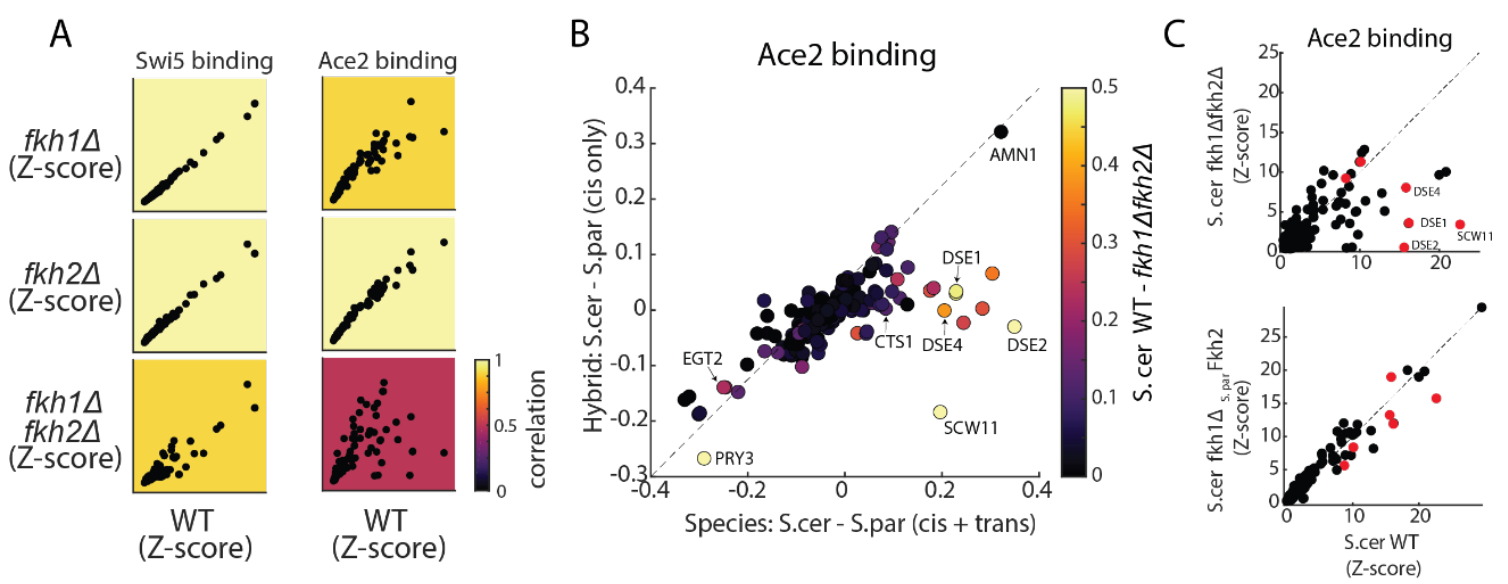

D s. cerevisiae:

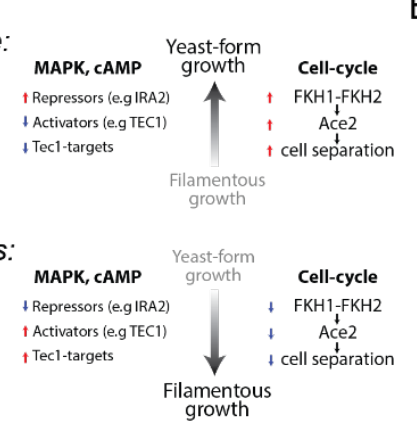

E

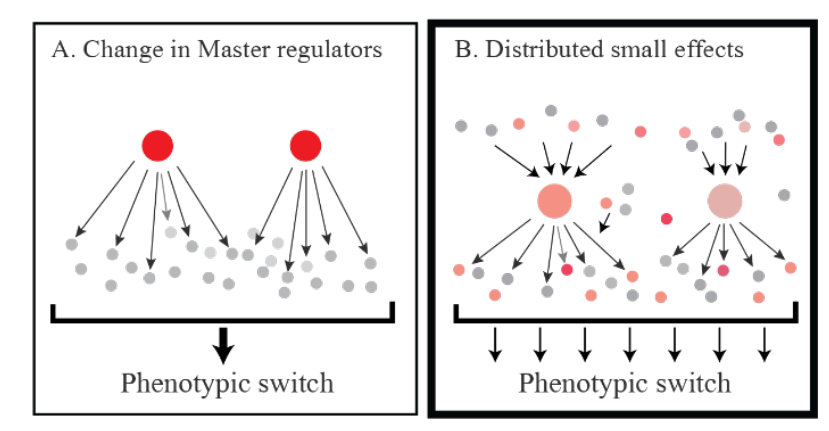

Fig 5. Fkh1 and Fkh2 directly mediates Ace2 binding to cell separation genes (A) Ace2 binding is affected by Fkh1 and Fkh2. Shown are normalized sum of signal on promoters for Swi5 and Ace2 in WT S. cerevisiae compared to $f k h 1 \Delta, f k h 2 \Delta$ and $f k h 1 \Delta f k h 2 \Delta$. Color represents correlation of top 100 promoters to WT. (B) Cis and trans effects on Ace2 binding. Scatter plot of relative Ace2 binding differences between species (x-axis) to within hybrid (y-axis). Cis effects are therefore along the diagonal while trans effects deviate from it. Color represents the differences in Ace2 binding between S. cerevisiae WT to $f k h 1 \Delta f k h 2 \Delta$. Note that cell separation genes show a strong trans effect and are strongly affected by $f k h 1 \Delta f k h 2 \Delta$. (C) Expression of S. paradoxus FKH2 in $S$. cerevisiae restores Ace2 binding. Scatter plots of normalized sum of signal on promoters of Ace2 in S. cerevisiae WT vs $f k h 1 \Delta f k h 2 \Delta$ (top), and in S. cerevisiae WT vs. fkh1 $1 \Delta$ expressing S. paradoxus FKH2 (right). Red dots represent cell separation genes. (D) overview of inter-species regulatory differences. Expression variations across different regulatory levels, affecting both filamentous regulation and cell cycle regulation, supporting the respected growth mode. Red and blue arrows indicate higher or lower expression, respectively.(E) - Model of evolutionary adaption of complex phenotypes: While mutations that alter activity or expression of a master regulator could activate the cellular (A), we suggest that weak distributed effects accumulate in different layers of the regulatory network, driving and stabilizing the different response (B). 


\section{Supplementary figures}

Figure S1

A
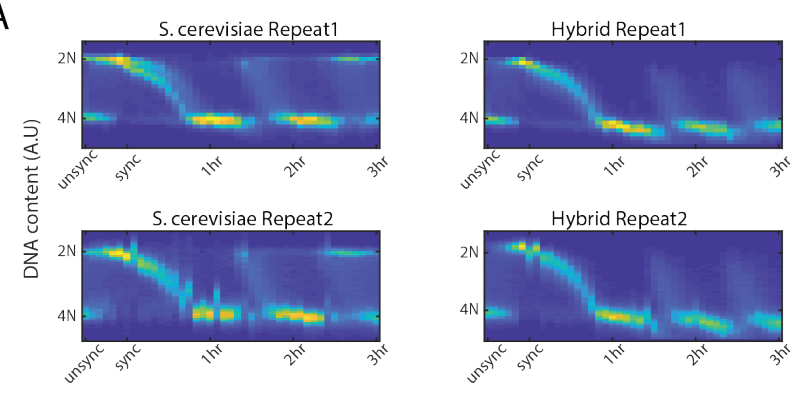

Species

B

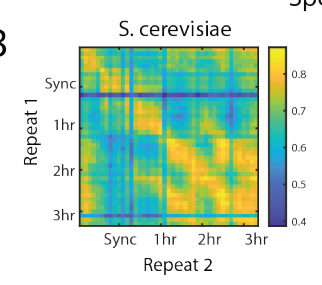

C
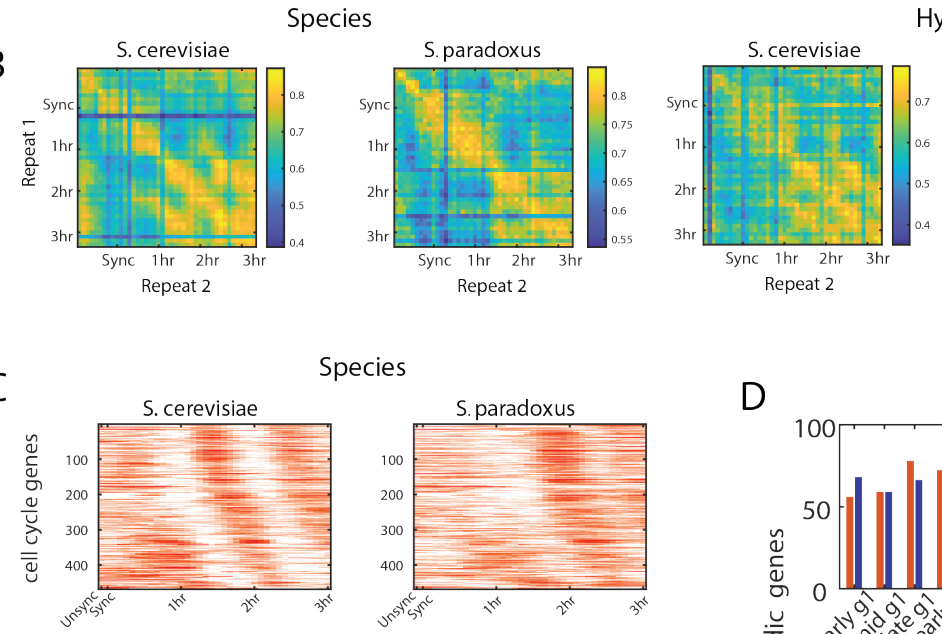

Hybrid
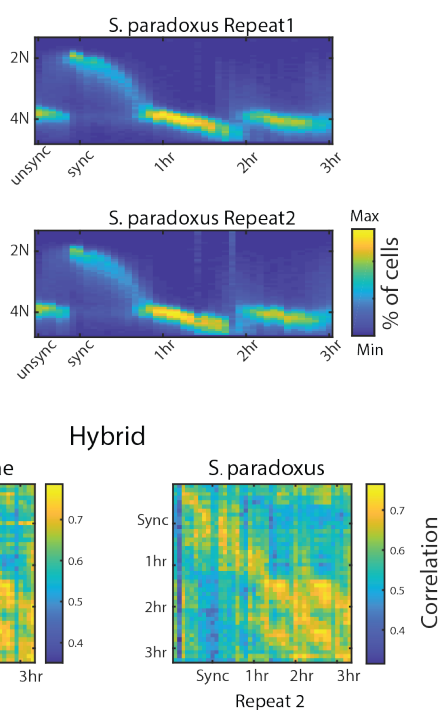

Species

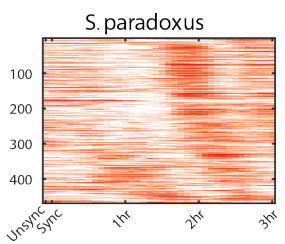

D

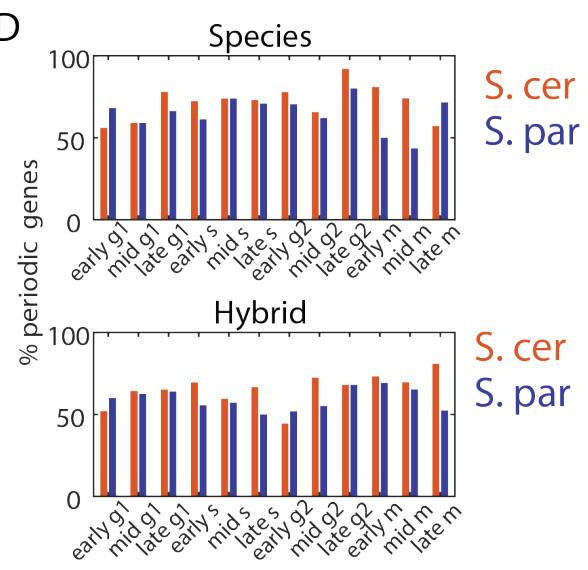

$E$
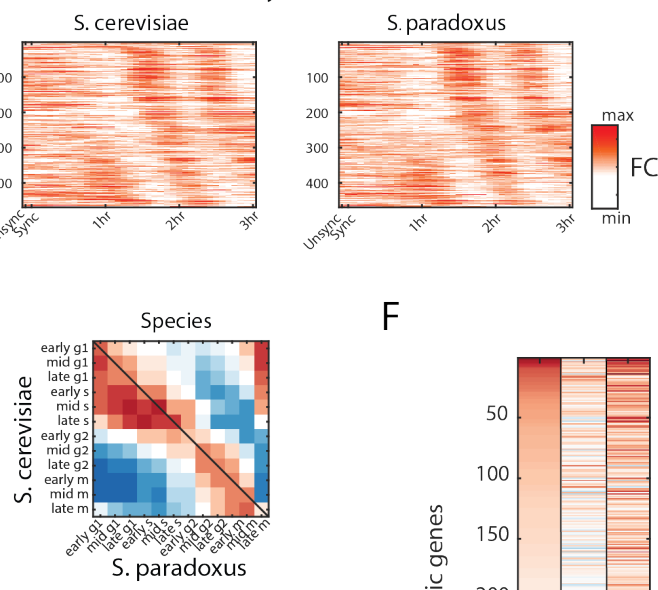

S. cer

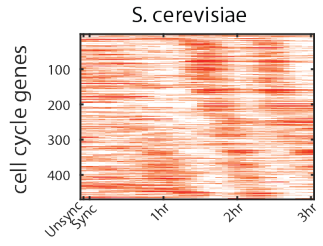

$\mathrm{F}$
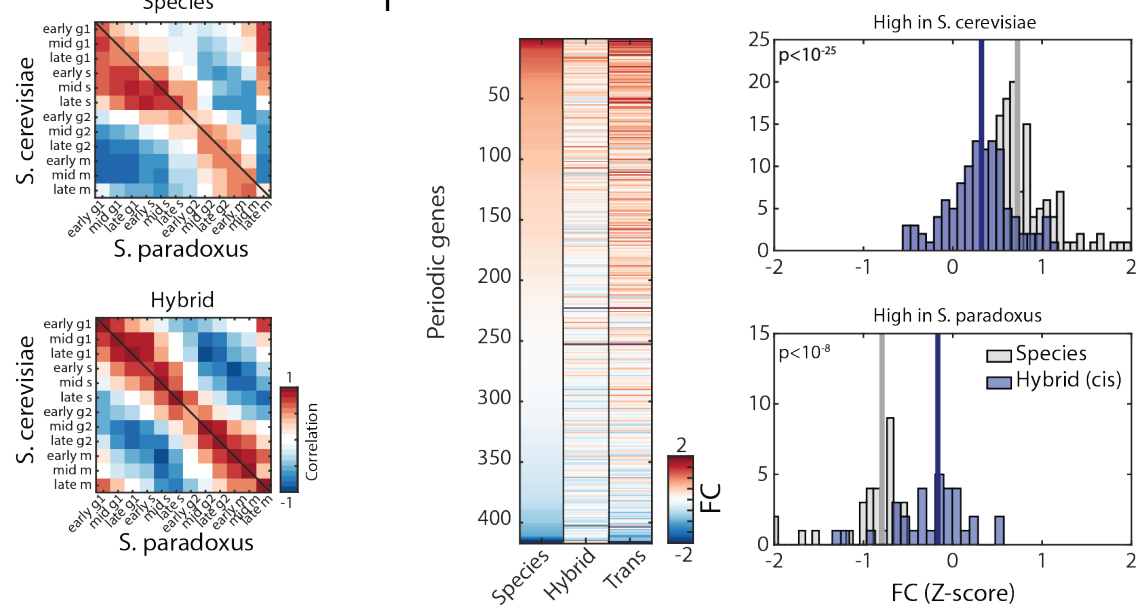
S1 Fig. Monitoring the gene expression program along the cell cycle. (A) DNA staining profiles along the cell cycle in the two repeats of S. cerevisiae, S. paradoxus and the hybrid. (B) Correlation matrices of periodic transcripts (top 500) between the two repeats. Note high correlation along the diagonal and high correlation between first and second cycle. (C) Expression of periodic transcripts. Each gene was normalized to its median expression, plotted are all periodic genes ordered by their expression peak time. (D) Bar graphs showing the percentage of genes with significant correlation to the respected module (corrected $p$-value $<0.05$, rho $>0.4$ ). (E) Periodic co-expression of cycling genes. Interspecies (left) and within hybrid (right) correlations between mean expression of cell cycle modules, classified to 12 groups. Note high correlations between early G1 to late genes in S. paradoxus, indicating rapid activation of S-phase genes. (F) Fold changes in expression levels of periodic genes. Left: shown are fold change (FC) in absolute levels at expression peak between species, hybrid and trans (Species-hybrid). Genes ordered by high expression differences in S. cerevisiae. Right: distribution of top changing genes in S. cerevisiae (top) and S. paradoxus (bottom). 
A

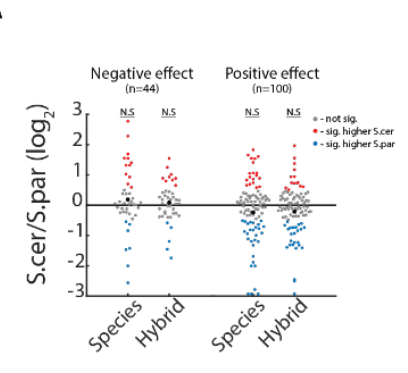

C

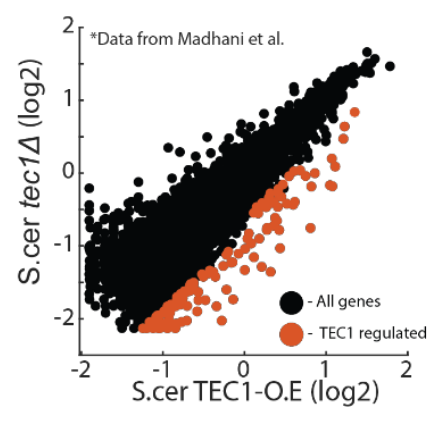

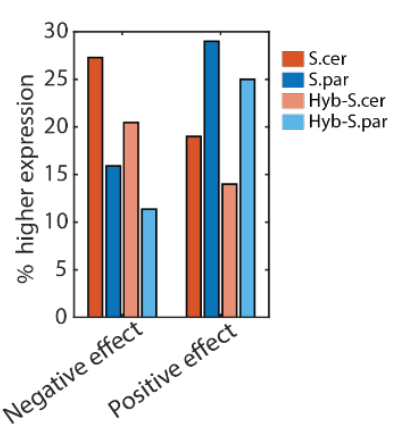

D
B

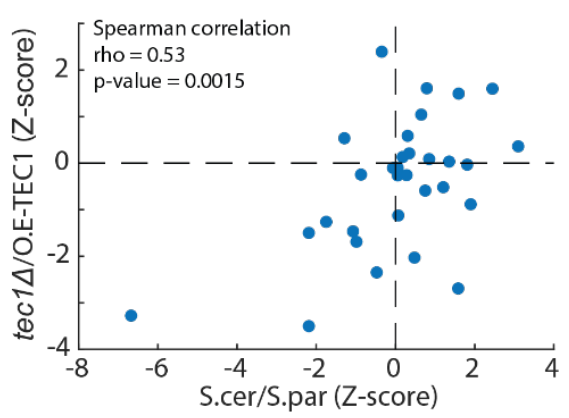

$E$
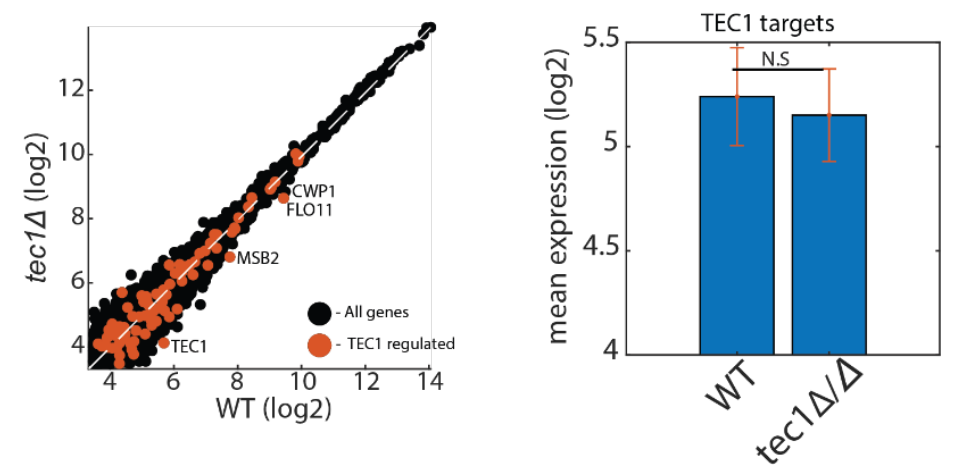

S. parphd1 $1 \Delta / \Delta$

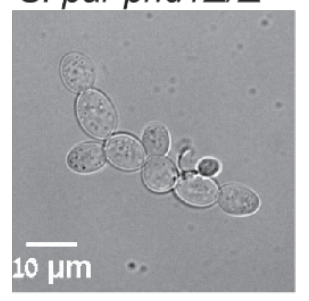

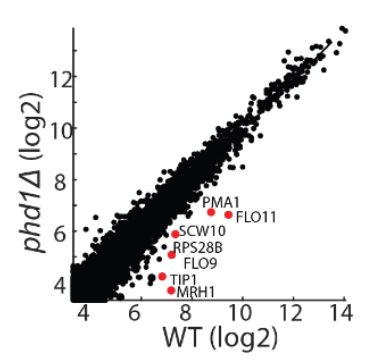

S. par $\operatorname{rim} 101 \Delta / \Delta$
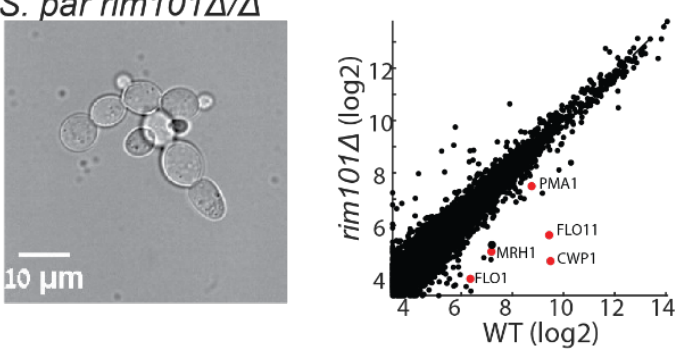

S2 Fig. Expression of filamentous regulators. (A) Expression variations in genes that have reported negative $(n=44)$ or positive $(n=100)$ effect on filamentous growth. Left: Shown are average fold-change (FC) distributions across all timepoints. Red and blue dots indicate significantly higher expression in S. cerevisiae and S. paradoxus, respectively. Right: percentage of differential expressed genes in each group, note bias in cis. (B) Expression variations between species is similar to expression changes between $S$. cerevisiae in yeast-growth or filamentous growth. Shown are FC values of genes in MAPK and CAMP-PKA pathway between species ( $x$-axis) to between tec1 $\triangle$ to TEC1-over expression (y-axis). Data is presented in Z-scores. (C) Determining Tec1-regulated genes. Genes were defined as the genes showing significant change between TEC1-over expression to tec1 $\triangle$. (D) Expression of Tec1-regulated genes in S. paradoxus WT and in tec $1 \Delta / \Delta$. (E) Phenotype and expression of $\operatorname{rim} 101 \Delta / \Delta$ and phd $1 \Delta / \Delta$ in S. paradoxus grown in YPD. 
Cells still exhibit filamentous growth, while cell-wall genes (such as FLO11, FLO9, CWP1) show lower expression.
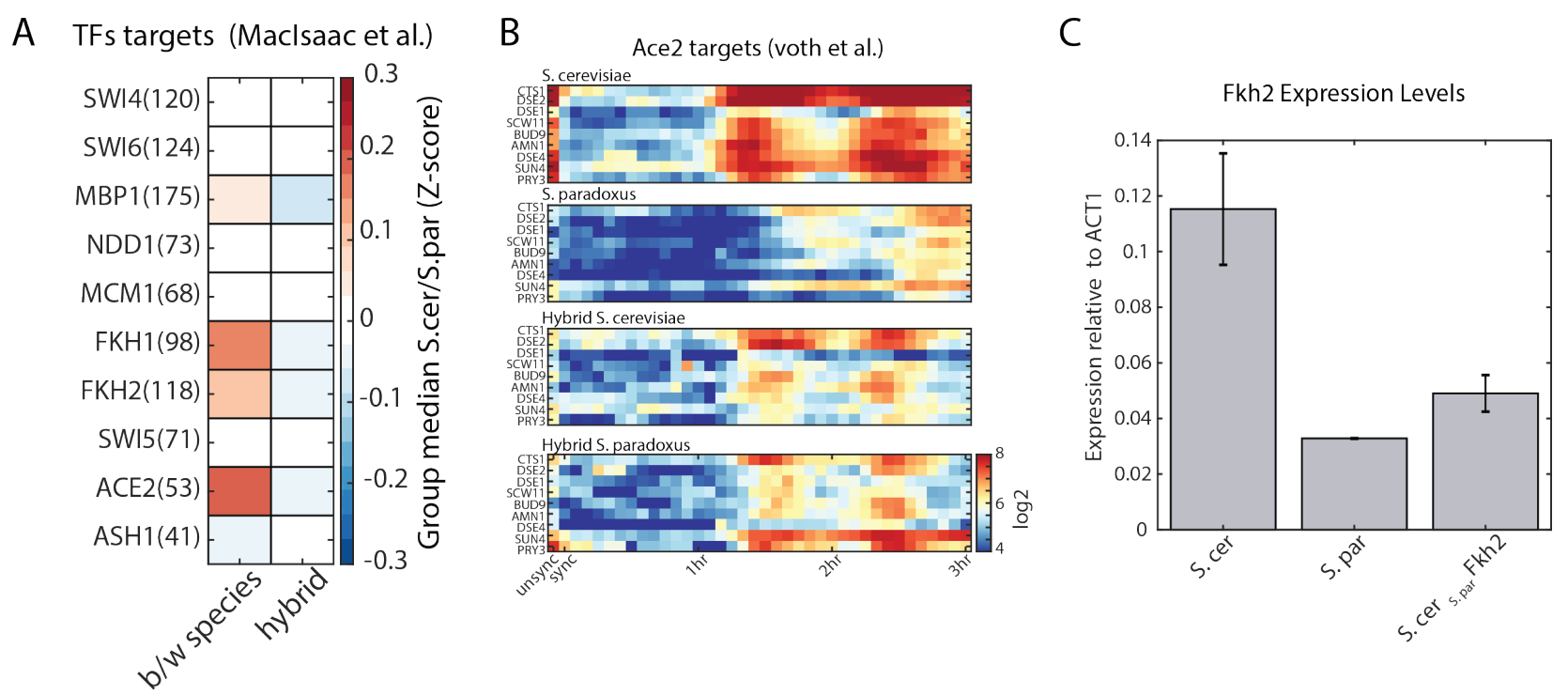

S3 Fig. Downregulation of ACE2 targets in S. paradoxus. (A) Median FC (indicated in Z-scores) of TFs targets defined by another dataset ([73]). Number indicates the number of targets genes in the respected group. (B) Absolute expression levels of Ace2 targets (as defined by [54]) along the cell cycle (C) FKH2 expression levels, as measured by RT-qPCR, in S. cerevisiae, S. paradoxus and S. cerevisiae expressing S. paradoxus FKH2. 
A Correlations between repeats

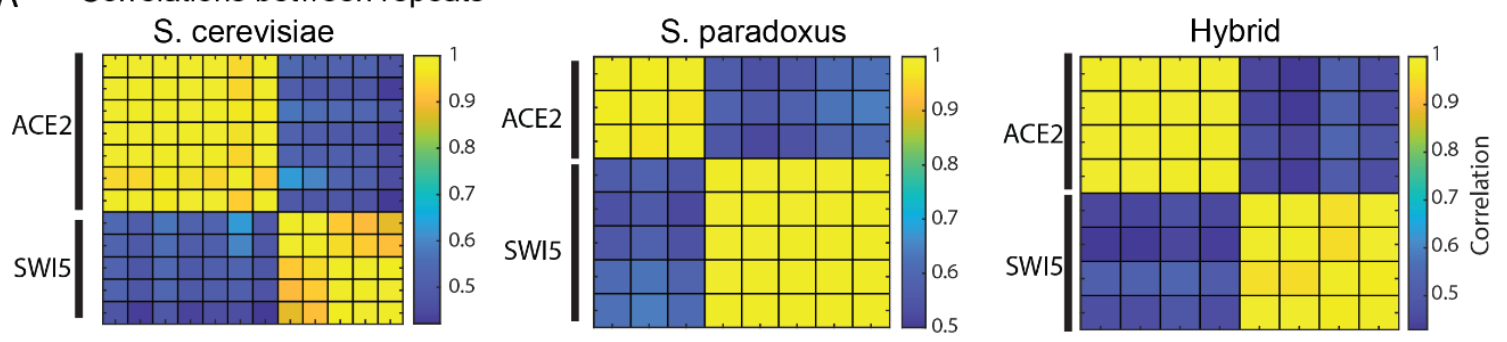

B

Meta-Gene profiles
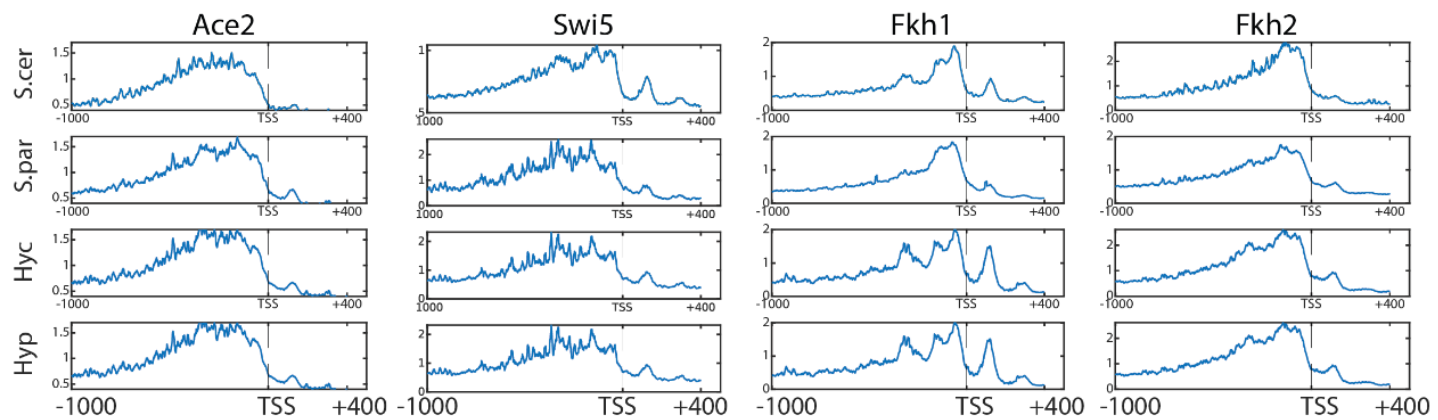

\section{Motif preferences}
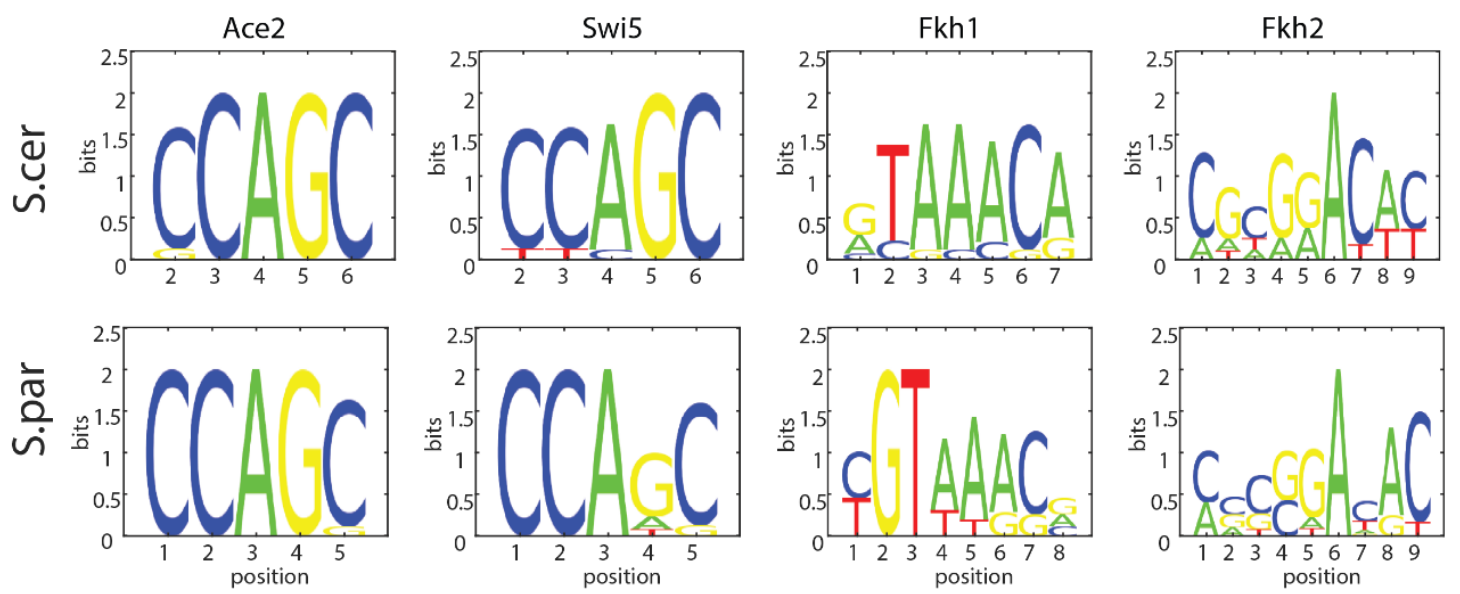

S4 Fig. Binding patterns of Ace2, Swi5, Fkh1 and Fkh2. (A) Pearson correlation matrices of normalized sum signal on promoters between repeats of Ace2 and Swi5, in S. cerevisiae, S. paradoxus and hybrid (S. cerevisiae genome). (B) Meta gene profiles of all TFs. All genes were aligned according to their transcription start site (TSS), and the signal was averaged across all genes. (C) Sequence logos representing the DNA-motif bound by each factor (See supplementary methods). Ace2, Swi5 and Fkh1 shows binding to their known DNA-motif. Fkh2 doesn't show a 
clear logo, though it did get among the top motifs it's known DNA motif (GTAAACA, not shown). Additional high scoring motifs in Fkh2 might result from interactions with other proteins.

A

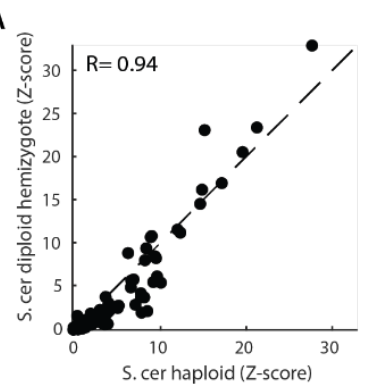

B
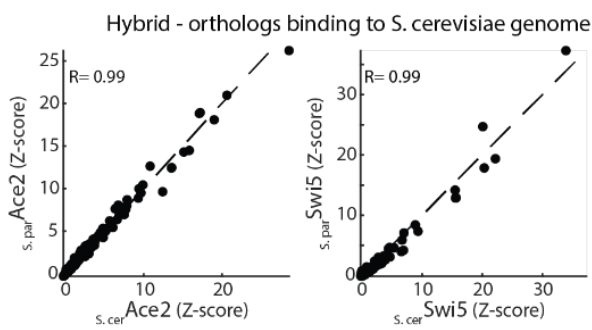

D

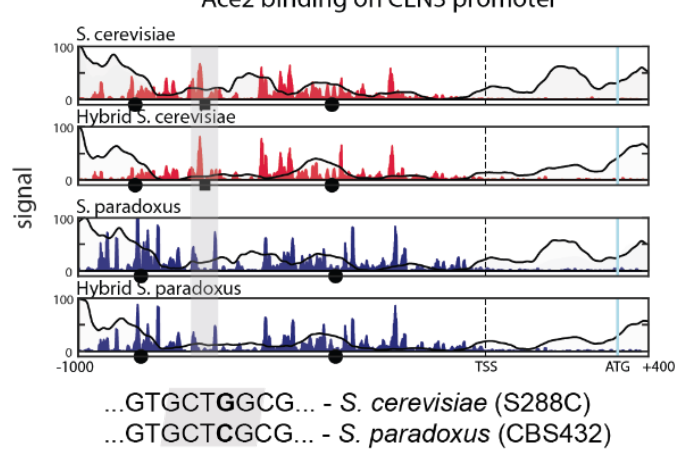

Ace2 - haploid vs diploid
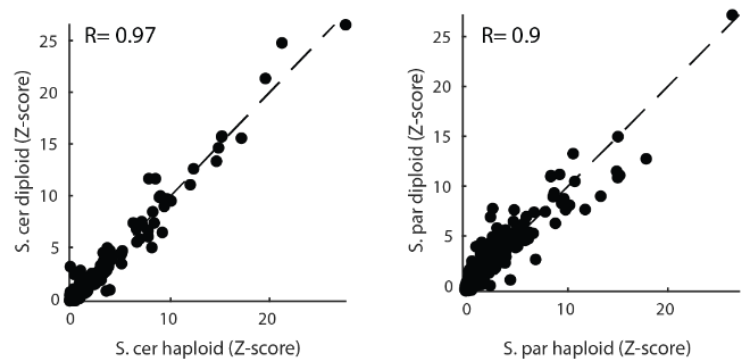

C

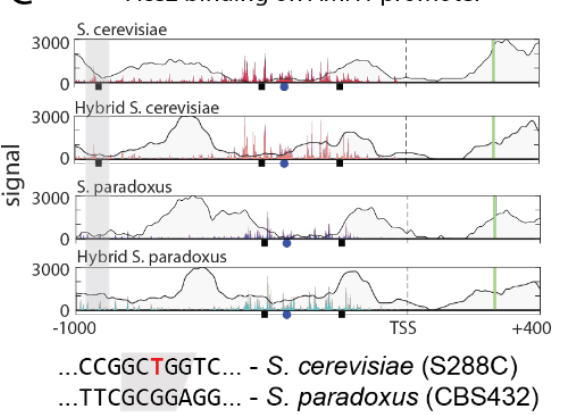

E
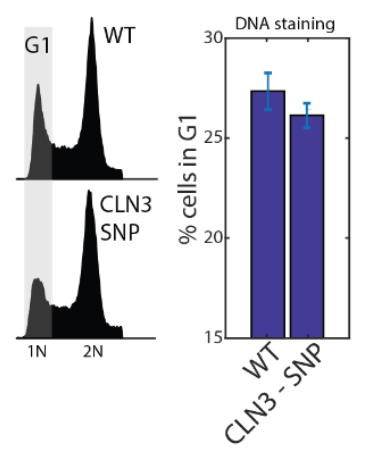

$\mathrm{F}$

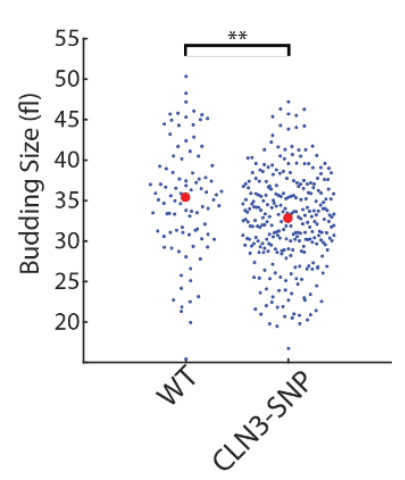

S5 Fig. cis and trans effects on Ace2 binding. (A) plotted is Ace2 normalized sum of signal on each promoter. Pearson correlation values of top 100 promoters are shown. Left: S. cerevisiae haploid vs S. cerevisiae hemizygote diploid expressing only one copy of ACE2. Middle: S. cerevisiae haploid vs diploid. Right: S. paradoxus haploid vs diploid. (B) Normalized sum of signal on each promoter of Ace2 and Swi5 in the hybrid, comparing binding of the two orthologs genes of each factor. (C) Ace2 binding on AMN1 promoter. (D) Ace2 binding on CLN3 promoter. Shown are the sequence changes leading to loss or gain of Ace2 binding site. (E) Left: DNA staining profile of S. cerevisiae strain carrying the mutation of S. paradoxus in CLN3 promoter. Right: quantification of \% cells 
with 1N DNA content. Shown are the mean and standard error of 4 repeats. (F) quantification of G1 duration in daughter cells in live microscopy (see methods). Reduced budding size indicates reduces cell size control. Asterisks represent significant p-value (two-sample t-test).

Figure S6

A

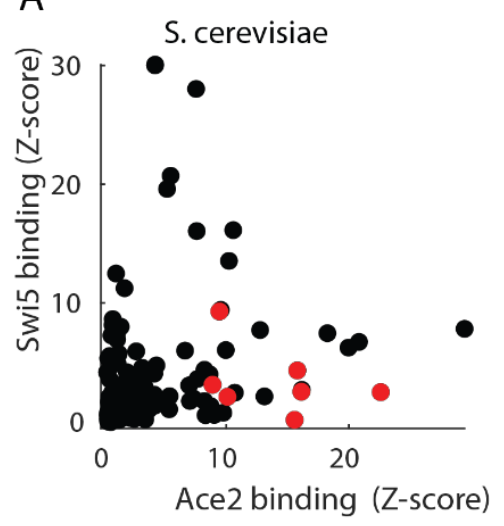

C

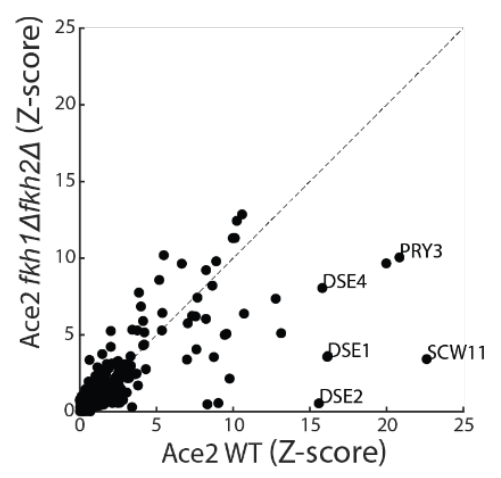

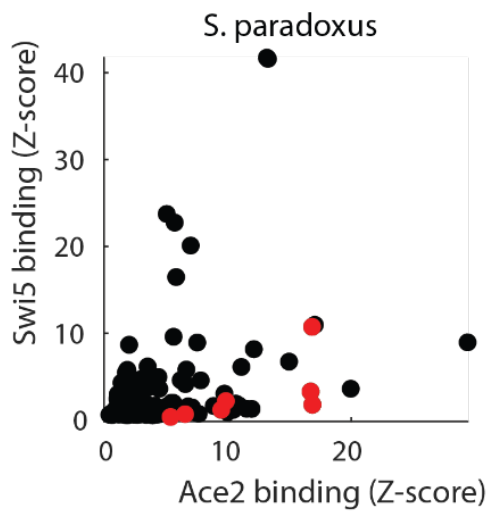

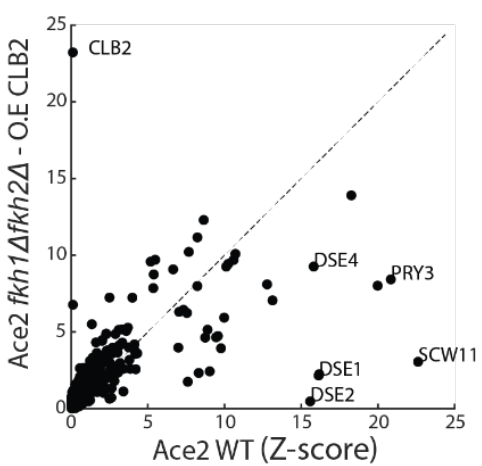

B
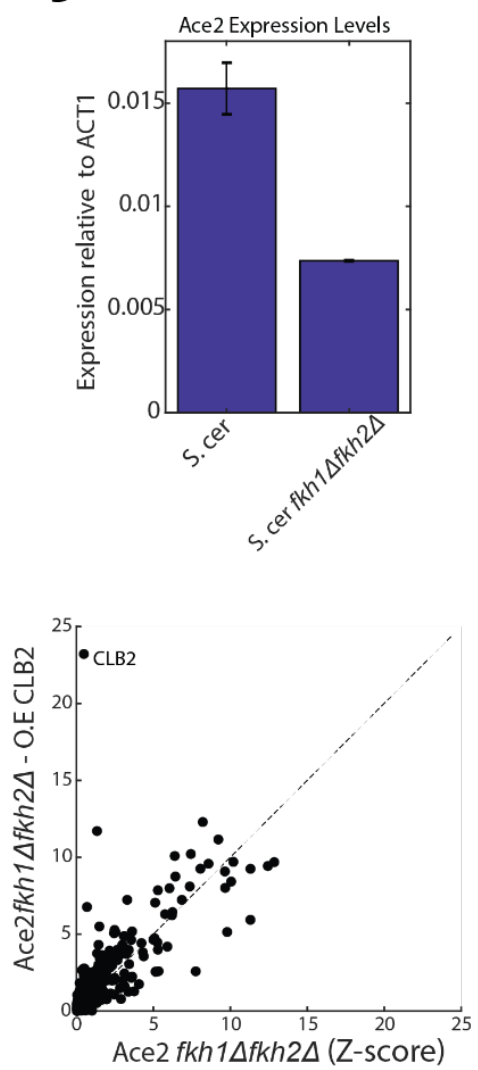

S6 Fig. Fkh1 and Fkh2 directly mediates Ace2 binding to cell separation genes. (A) sum signal on promoter of Ace2 and Swi5 in S. cerevisiae and S. paradoxus. Red dots indicate cell separation genes. (B) ACE2 relative expression levels in WT and in $f k h 1 \Delta f k h 2 \Delta$, as measured by RT-qPCR. (C) over expression of CLB2 doesn't restore Ace2 binding to cell separation genes. Shown are sum signal on promoters of Ace 2 in WT vs $f k h 1 \Delta f k h 2 \Delta$, WT vs $f k h 1 \Delta f k h 2 \Delta$ CLB2-O.E, and $f k h 1 \Delta f k h 2 \Delta$ vs $f k h 1 \Delta f k h 2 \Delta$ CLB2-O.E. 